ESTIMATION OF NATURAL STREAMFLOW CHARACTERISTICS

IN WESTERN COLORADO

By James E. Kircher, Anne F. Choquette, and Brian D. Richter

U.S. GEOLOGICAL SURVEY

Water-Resources Investigations Report 85-4086

Prepared in cooperation with the

U.S. BUREAU OF LAND MANAGEMENT

Lakewood, Colorado

1985 


\section{UNITED STATES DEPARTMENT OF THE INTERIOR}

DONALD PAUL HODEL, Secretary

GEOLOGICAL SURVEY

Dallas L. Peck, Director

For additional information write to:

District Chief

U.S. Geological Survey

Box 25046, Mail Stop 415

Denver Federal Center

Lakewood, CO 80225
Copies of this report may be purchased from:

Open-File Services Section Western Distribution Branch U.S. Geological Survey Box 25425, Denver Federal Center Lakewood, CO 80225

Telephone: (303) 236-7476 
Page

Glossary--0-0

Abstract--

Introduction--

Description of study area--

Previous studies---

Purpose and scope--

Development of estimating relations--

Analytical technique--

Data used-- A

Streamflow records-- 4

Basin and climatic characteristics- 6

Hydrologic regions--- 6

Regression relations--

Application of regression relations--

Gaged sites-- 16

Ungaged sites--

Limitations and accuracy-

Summary and conclusions--

References cited--

\section{ILLUSTRATIONS}

Plate 1. Map showing location of hydrologic boundaries and streamflowgaging stations used to define the regions in western Colorado--

Figures 1-3 Graphs showing:

1. Basin drainage area and length of record for the gaging stations used in the regression analyses----

2. Range and distribution of basin characteristics used in regression relations for each hydrologic region-

3. Mean standard error of regression estimates for selected flow characteristics in each hydrologic region--

\section{TABLES}

Tables 1-4. Gaging-station records used to determine regression relations for the:

1. Mountain region-- 7

2. Rio Grande region--- 10

3. Southwest region--

4. Northwest region-- 13

5-8. Summary of regression relations for the:

5. Mountain region- 17

6. Rio Grande region- 18

7. Southwest region-- 19

8. Northwest region- 20 


\section{GLOSSARY}

Water-resource terms are defined in the GLOSSARY and are italicized when first used in this report.

basin characteristics.--Physical and climatic conditions of a basin that are used in the regression models to predict streamflow. Basin characteristics defined in the final regression models include:

drainage area, in square miles, computed by planimeter from the best available U.S. Geological Survey topographic maps.

mean basin slope, in feet per feet, based on the average of 25 or more slope values taken at equal-spaced grid points on 1:50,000-scale U.S. Geological Survey topographic maps (Lystrom and others, 1978).

mean basin elevation, in feet above the National Geodetic Vertical Datum of 1929, determined from 25 or more equal-spaced grid points from 1:50,000-scale U.S. Geological Survey topographic maps.

mean annual precipitation, in inches to the nearest 0.1 inch, the average of 25 or more mean annual precipitation values at equal-spaced grid points on an isohyetal map (Colorado Climate Center, 1984).

flood-frequency relation.--A graph showing the probability that the annual peak discharge for a given year will exceed the indicated amount.

flow-duration series.-Daily flows that are, on the average, equaled or exceeded $10,25,50,70$, or 90 percent of the time. Flow values were interpolated from duration curves (U.S. Geological Survey, 1979) of daily flows during the period of gaged record.

gaging station.--A particular site on a stream or other body of water where systematic observations of gage height, discharge, or water quality constituents (or any combination of these) are obtained.

log-Pearson Type III frequency distribution.--A statistical distribution used in flood-frequency analysis, which is described by three parameters: mean, standard deviation, and coefficient of skewness of the logarithms of the sample observations (see U.S. Water Resources Council, 1981).

mean annual discharge.--The mean of a series of annual water discharges, in cubic feet per second.

mean monthly discharge.--The mean of a series of monthly mean discharges for each calendar month.

mean standard error.--The mean of positive and negative departures of observed values from values predicted by the regression relation; a measure of how well observed data agree with the regression estimates.

minimum 7-day discharge.--The minimum mean discharge occurring over a period of seven consecutive days, with average recurrence intervals of 2,10 , and 50 years. Also referred to as low flows throughout this report. 
maximum 7-day discharge.--The maximum mean discharge occurring over a period of 7 consecutive days, with average recurrence intervals of $2,10,50$, and 100 years. Also referred to as high flows throughout this report.

multiple-regression relations.--A statistical technique by which a relation between a dependent variable and two or more independent variables can be derived. The result usually is expressed as a regression equation.

natural flow.--Stream discharge that is not significantly affected by human land use or water use, such as flow diversion, regulation, or vegetative alteration.

normalize.--To transform a variable so that the probability distribution of the transformed variable approximates a normal distribution.

orographic effect.--The lifting of moisture-laden air over a high barrier such as a mountain range resulting in a consequent release of precipitation.

parameter.--A descriptive measure of a population, such as a mean, a measure of variability, or a regression coefficient.

peak discharge.--Instantaneous maximum discharges that were exceeded on the average of once every $2,5,10,25,50,100,200$, and 500 years.

recurrence interval.--The mean interval of time, in years, within which a given flood discharge will be exceeded once.

regression.--A statistical technique applied to paired data to determine the degree of mutual association between a dependent variable and one or more independent variables.

residual.--The discrepancy between measured streamflow and the regression estimate of flow at that site.

\section{METRIC CONVERSION FACTORS}

Inch-pound units used in this report may be converted to International System of Units (SI) by using the following conversion factors:

To convert inch-pound unit

cubic foot per second $\left(\mathrm{ft}^{3} / \mathrm{s}\right)$

inch (in.)

foot $(f t)$

square mile $\left(\mathrm{mi}^{2}\right)$
Multiply by

0.02832

25.40

0.3048

2.590
To obtain metric unit

cubic meter per second

millimeter

meter

square kilometer 


\title{
ESTIMATION OF NATURAL STREAMFLOW CHARACTERISTICS IN WESTERN COLORADO
}

By James E. Kircher, Anne F. Choquette, and Brian D. Richter

\begin{abstract}
Regression relations were determined for estimating mean annual discharge, mean monthly discharge, flow-duration series, peak discharge, and minimum and maximum 7-day discharges for natural-flow streams in western Colorado. The techniques can be applied to both gaged and ungaged streams. Multiple regression analyses were used to determine the best regression relations for each of the streamflow characteristics. Separate regression relations were developed for each of four hydrologically distinctive regions in the study area. The mean standard errors associated with the regression relations generally were less than 100 percent except for the low-flow relations which had standard errors ranging from 62 to greater than 200 percent. Basin drainage area, mean annual precipitation, mean basin elevation, and mean basin slope are used in the regression relations to determine flow characteristics of streams in the study area.
\end{abstract}

\section{INTRODUCTION}

\section{Description of Study Area}

The study area, located in western Colorado (p1. 1), extends westward from the Front Range in the northeast and the Sangre de Cristo Mountains in the southeast to the Colorado-Utah state line. The eastern border of the study area follows the 7,500-ft elevation contour in the Platte River basin and the 9,500-ft contour in the Arkansas River basin and coincides approximately with the boundary between the Southern Rockies and the Great Plains physiographic provinces (Fenneman, 1931).

In the eastern part of the study area, the major landforms are the Rocky Mountains which range in elevation from about 7,500 to more than 14,000 ft. The western part of the study area consists predominantly of broad plateaus and mesas; elevations range from about 5,000 to 7,500 ft except for Grande Mesa, 11,000 ft, the Uncompahgre Plateau, 10,000 ft, and the Roan Plateau, $8,500 \mathrm{ft}$. Annual precipitation ranges from less than 7 in. at lower elevations to more than 60 in. in the high mountains (Colorado Climate Center, 1984). 
Mountains in the eastern part of the study area are underlain primarily by Precambrian igneous and metamorphic rocks and Paleozoic sediments. The San Juan Mountains, in the south-central part of the region, consist predominantly of Tertiary volcanics. Rock types in the western part of the study area consist predominantly of sandstones, siltstones, shales, and conglomerates of Cretaceous and Tertiary age.

The North Platte, South Platte, Arkansas, and Rio Grande Rivers drain the eastern part of the study area. All rivers in the western part drain into the Colorado River and include: (1) The Yampa and White Rivers in the northwest, (2) the main stem of the Colorado River and the Gunnison River in the westcentral, and (3) the Dolores, Animas, and San Juan Rivers in the southwest.

\section{Previous Studies}

Several previous reports have presented methods for estimating flow characteristics of natural basins in the study area. A series of reports in the mid- to late-1960's defined flood-frequency relations for the South Platte (Matthai, 1968), Arkansas (Patterson, 1964), Rio Grande (Patterson, 1965), and Colorado (Patterson and Somers, 1966) River basins. McCain and Jarrett (1976) used improved techniques for estimating flood frequency and additional streamflow records to estimate flood characteristics for streams in Colorado. Livingston (1970) developed relations for a range of flow characteristics, similar to those included in this study, for the mountains of Colorado. For the mountain region of Colorado, Hedman and others (1972) improved the accuracy of some of Livingston's (1970) models by relating measurements of channel geometry to mean annual and peak flows.

This study differs from these previous studies in areal coverage, range of flow characteristics being estimated, number of gaging stations, lengths of gaged-runoff records, and methods used to define the regression relations. The reports by Patterson (1964, 1965), Patterson and Somers (1966), and Matthai (1968) are based on regional flood-frequency analyses derived using the index-flood method (Dalrymple, 1960). Subsequent reports are based on flow characteristics derived from a log-Pearson Type III frequency distribution. Compared to most of the previous studies, this study is based on up to 13 additional years of gaged-flow measurements, a recently revised arealprecipitation map (Colorado Climate Center, 1984), and improved statistical techniques for model selection.

\section{Purpose and Scope}

This study provides methods for estimating flow characteristics of natural-flow streams in western Colorado. Equations were developed for a total of 33 flow characteristics, which include mean annual and mean monthly discharge, flow-duration series, peak discharge, and minimum and maximum 7-day discharges of recurrence intervals ranging from 2 to 100 years. The procedures for estimating flow differ depending on whether the estimate is for a gaged or ungaged site, and whether the drainage area above the site crosses hydrologic region boundaries or state lines. 
The study was conducted in cooperation with the U.S. Bureau of Land Management to provide a methodology to assess streamflow characteristics for land use, planning, impact assessment, and identifying potential project locations.

The regression relations were developed using multiple regression analyses and are based on 10 or more years of streamflow records for 264 stations located in and adjacent to the study area (pl. 1). The study area was divided into four hydrologic or streamflow-characteristic regions to remove geographic bias resulting from differences in basin physiography and climate. Separate regression relations were developed for each of these four regions.

\section{DEVELOPMENT OF ESTIMATING RELATIONS}

\section{Analytical Technique}

The regional regression relations discussed in this report are regression equations that relate streamflow characteristics to easily measured drainage basin and climatic measurements. The streamflow characteristic being estimated, the dependent variable, is determined from a selected set of basin characteristics, the independent variables. The expected accuracy of the regression estimates is indicated by the difference between the estimates and the gaged streamflow data.

Dividing an area into smaller subregions may reduce the variability of streamflow from site to site and result in more accurate streamflow estimates from regression equations. Regional analysis is based on the spatial variation of streamflow due to regional differences in the physical characteristics that directly or indirectly affect streamflow. Accuracy of the resulting streamflow regression relations for each hydrologic region is limited by: (1) The accuracy with which basin and climatic characteristics can be measured; (2) the difficulty in describing and measuring more variable or complex factors, such as vegetation water use, soil depth, and soil permeability that affect streamflow; and (3) the adequacy of the selected regression models to describe the hydrologic system.

Logarithmic transformations were performed on all streamflow and basin characteristics prior to the regression analyses. These data were transformed in order to: (1) Normalize the variables and residuals; (2) obtain a constant variance of the residuals about the regression line; and (3) obtain linear relations between dependent and independent variables. All of these specifications are needed to meet the statistical assumptions of regression analyses and to derive unbiased regression estimates. 
The multiple-regression relations based on logarithmic transformations of variables are of the form:

$$
\log Y=\log B_{0}+B_{1} \log X_{1}+B_{2} \log X_{2}+\ldots+B_{n} \log X_{n}
$$

or, taking antilogs,

$$
y=B_{0} x_{1}{ }^{B_{1}} x_{2}{ }^{B_{2}} \ldots x_{n}{ }^{B_{n}}
$$

where $\quad Y=$ streamflow characteristic (dependent variable), $\mathrm{B}_{0}, \mathrm{~B}_{1}, \mathrm{~B}_{2}, \mathrm{~B}_{\mathrm{n}}=$ regression coefficients,

$\mathrm{X}=$ basin or climatic characteristic (independent variable) upstream from the site for which the estimate is being made, and

$\mathrm{n}=$ number of basin and climatic characteristics in the model.

The regression coefficients are determined by regression analyses of information collected at gaged sites.

\section{Data Used}

Streamflow Records

Data from continuous-record gaging stations (pl. 1) were used to develop the regional streamflow regression relations. The continuous-record stations are operated by the U.S. Geological Survey in cooperation with other Federal and state agencies. The records used in this study included data collected through September 1981. Of the 810 past or present gaging stations located in the study area (see Richter and others, 1984), 264 stations were selected; these stations had 10 or more years of gaged record and were located in basins that were largely unaffected by urbanization or man-made structures, such as reservoirs or diversions. Stations were omitted if more than 10 percent of the basin's area was irrigated by upstream diversions or the amount of water in reservoir storage exceeded more than 10 percent of the volune of mean annual discharge. Plots of drainage areas versus lengths of record for the stations used in this study are shown in figure 1.

The streamflow characteristics determined are mean annual discharge, mean monthly discharge, flow-duration series, minimum 7-day discharge, maximum 7-day discharge, and peak discharge. The streamflow characteristics, which include those commonly needed for planning and design, were determined from the gaging-station records. The flow values are calculated parameters obtained from the U.S. Geological Survey National Water Data Storage and Retrieval System (WATSTORE) (see Hutchinson, 1975). All of the flow measurements are in cubic feet per second. Determination of annual discharge values was based on the October through September water year. Flow frequencies were estimated using the log-Pearson Type III frequency distribution (U.S. Water Resources Council, 1981). 


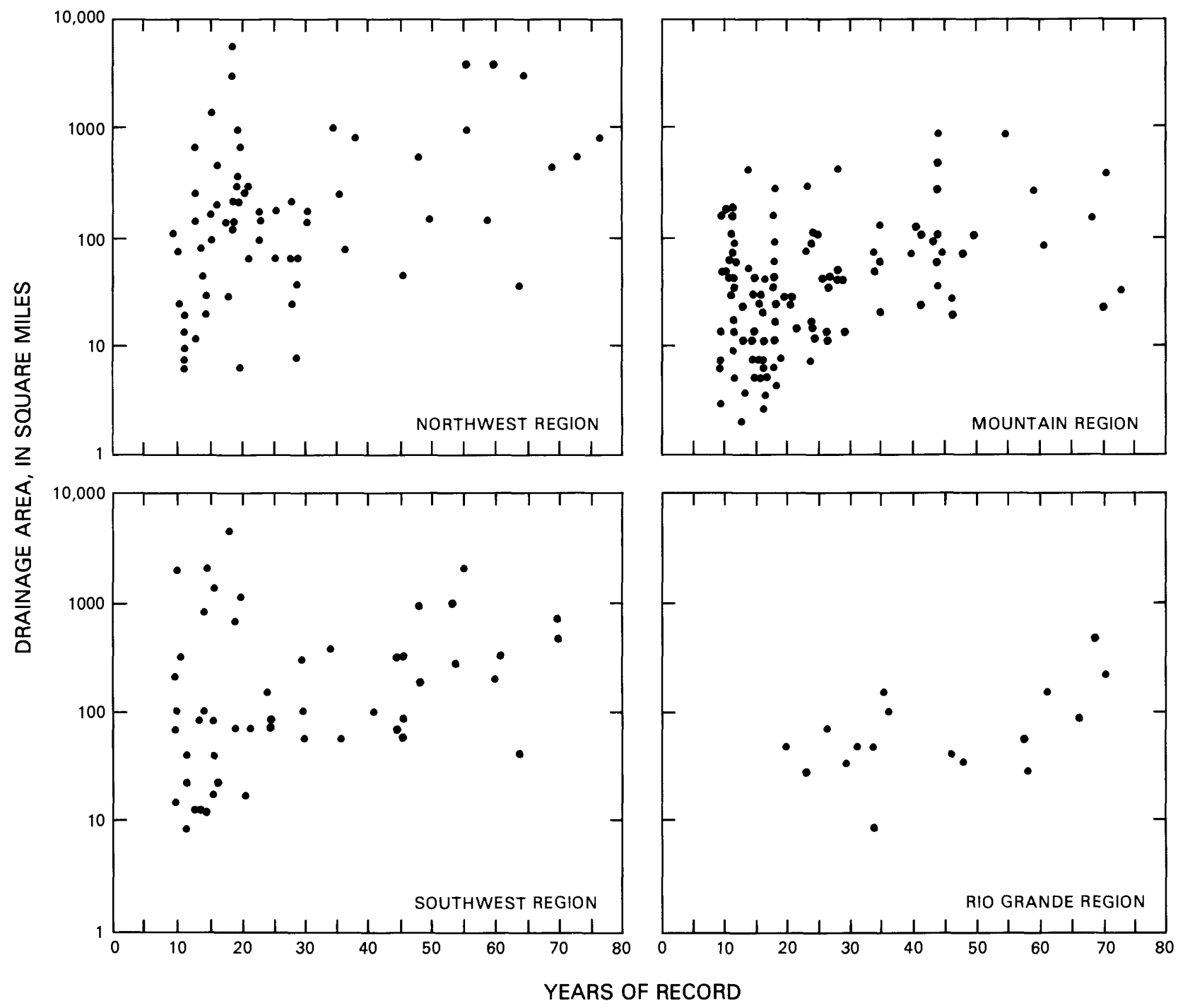

Figure 1.--Basin drainage area and length of record for the gaging stations used in the regression analyses. 
Values for each of the streamflow characteristics in the study basins (tables 1-4) are included in a comprehensive summary of data for all gaged basins in Colorado (Richter and others, 1984).

\section{Basin and Climatic Characteristics}

Based on the results of previous streamflow regionalization studies (for example Thomas and Benson, 1970; McCain and Jarrett, 1976) and consideration of physical characteristics that affect streamflow, a set of basin and climatic characteristics was tested initially in the regression analyses. The set of predictor characteristics that were initially tested in the regression analysis included the following physiographic, climatic, vegetation, and land use variables.

Physiographic characteristics:

1. Drainage area;

2. basin length;

3. mean basin aspect;

4. mean basin width;

5. mean basin slope;

6. channel length;

7. mean channel elevation;

8. mean channel slope;

9. mean channel aspect;

10. area covered by lakes and ponds; and

11. gaging-station elevation.

Climatic characteristics:

1. Mean annual precipitation;

2. maximum 24-hour precipitation intensity having a 2-year recurrence interval; and

3. mean annual January temperature.

Vegetation and land use characteristics:

1. Percentage of basin area covered by forest;

2. percentage of basin area used for agriculture;

3. percentage of basin area used for rangeland;

4. percentage of basin area classified as urban; and

5. percentage of basin area irrigated.

Richter and others (1984) define these characteristics in greater detail and summarize these basin characteristics for the study basins in this report.

\section{Hydrologic Regions}

Hydrologic regions were selected on the basis of an iterative procedure that delineated regional similarities in the relation between streamflow and basin characteristics. Initially, regression relations were developed for 
Table 1.--Gaging-station records used to determine regression relations for the mountain region [X, gaging station was used in the analysis]

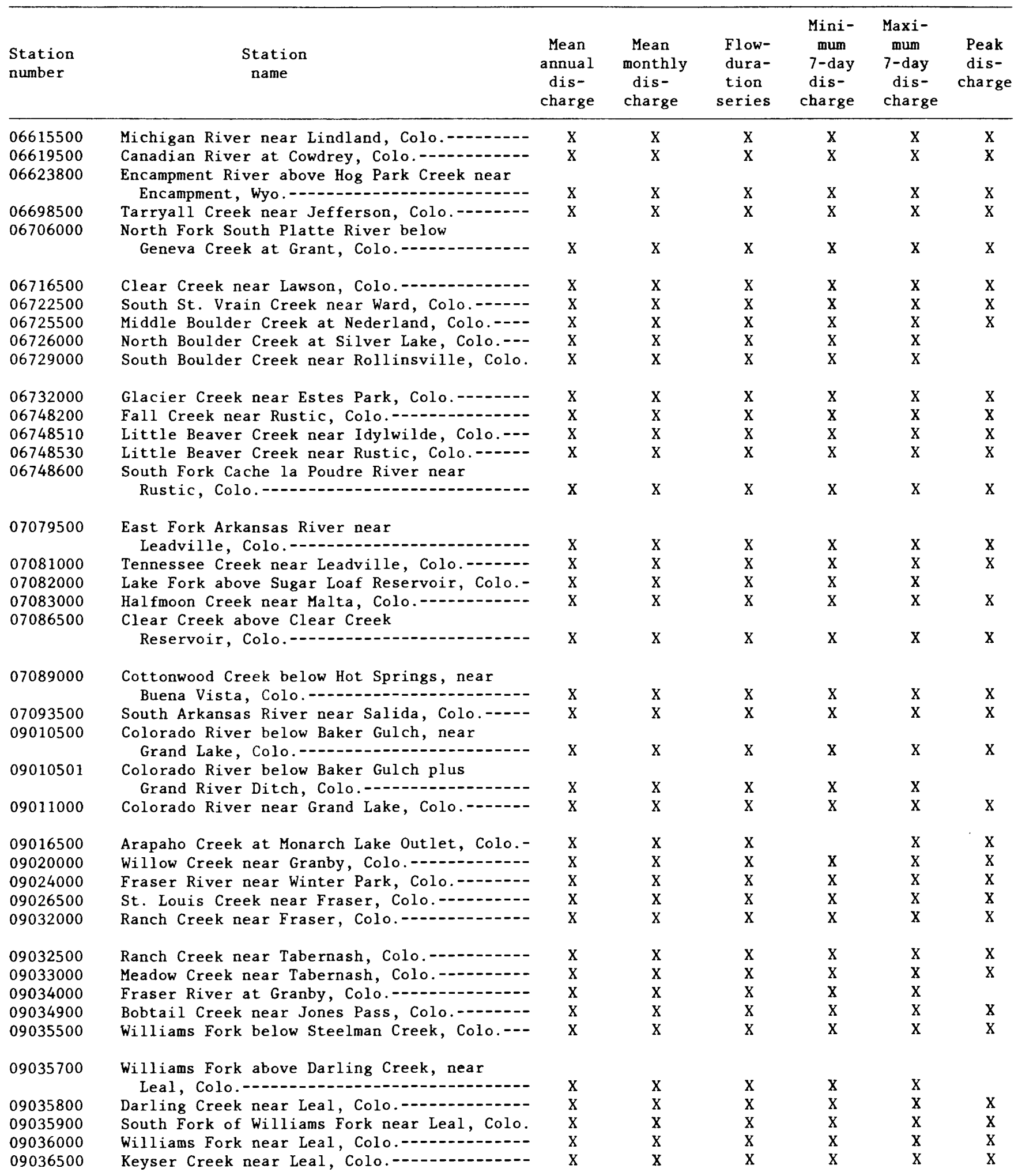


Table 1.--Gaging-station records used to determine regression relations for the mountain region--Continued

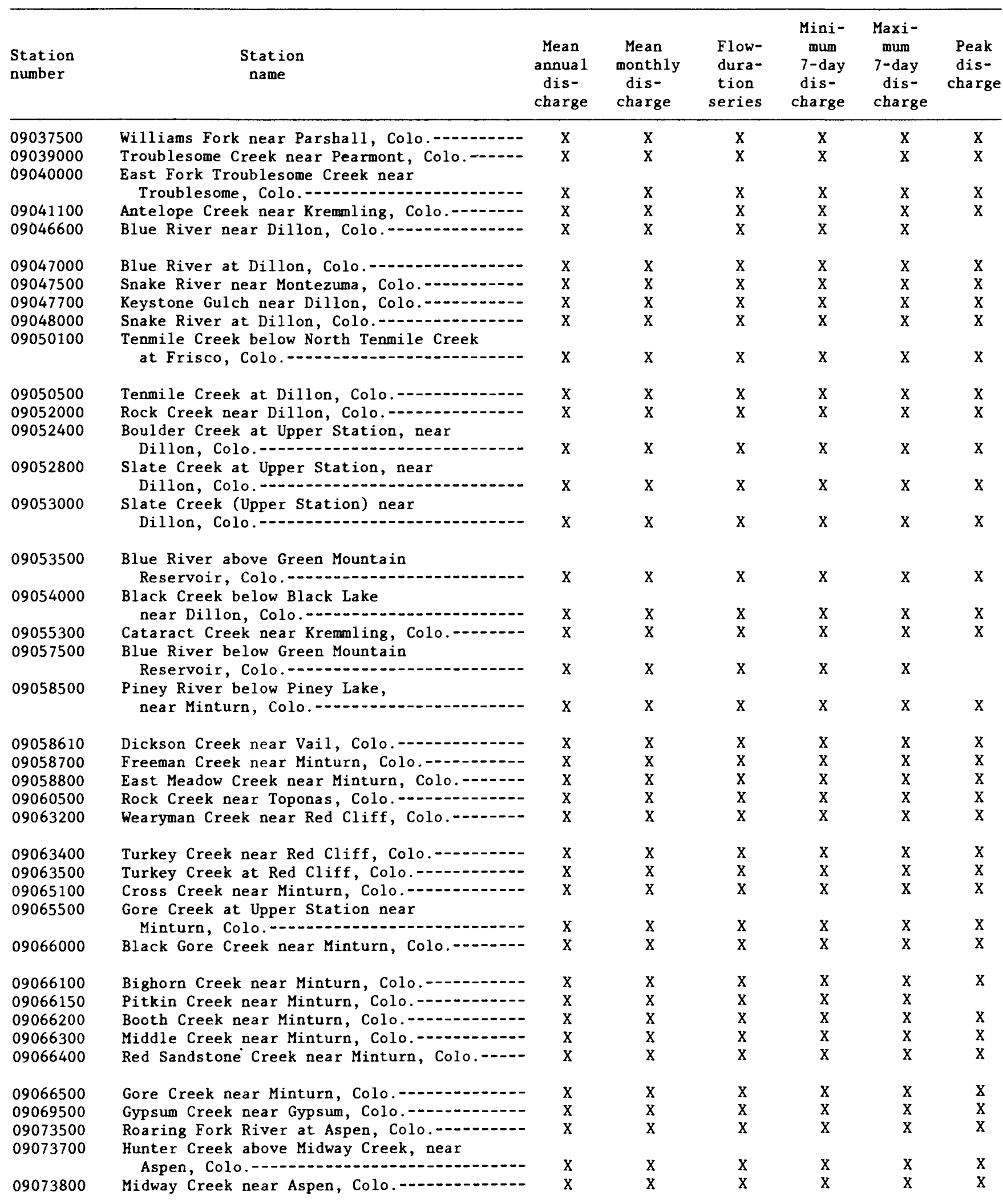


Table 1.--Gaging-station records used to determine regression relations for the mountain region--Continued

\begin{tabular}{|c|c|c|c|c|c|c|c|}
\hline $\begin{array}{l}\text { Station } \\
\text { number }\end{array}$ & $\begin{array}{l}\text { Station } \\
\text { name }\end{array}$ & $\begin{array}{c}\text { Mean } \\
\text { annual } \\
\text { dis- } \\
\text { charge }\end{array}$ & $\begin{array}{l}\text { Mean } \\
\text { monthly } \\
\text { dis- } \\
\text { charge }\end{array}$ & $\begin{array}{l}\text { Flow- } \\
\text { dura- } \\
\text { tion } \\
\text { series }\end{array}$ & $\begin{array}{c}\text { Mini- } \\
\text { mum } \\
7 \text {-day } \\
\text { dis- } \\
\text { charge }\end{array}$ & $\begin{array}{c}\text { Maxi- } \\
\text { mum } \\
\text { 7-day } \\
\text { dis- } \\
\text { charge }\end{array}$ & $\begin{array}{c}\text { Peak } \\
\text { dis- } \\
\text { charge }\end{array}$ \\
\hline 09073900 & No Name Creek near Aspen, Colo. & $\mathrm{X}$ & $\mathrm{x}$ & $\mathrm{X}$ & $\mathrm{X}$ & $\mathrm{X}$ & $\mathrm{X}$ \\
\hline 09074000 & Hunter Creek near Aspen, Colo. & $\mathrm{X}$ & $\mathrm{x}$ & $\mathrm{X}$ & $\mathrm{X}$ & $\mathrm{X}$ & $\mathrm{X}$ \\
\hline 09074800 & Castle Creek above Aspen, Colo...- & $\mathrm{X}$ & $\mathrm{x}$ & $\mathrm{X}$ & $\mathrm{X}$ & $\mathrm{X}$ & $\mathrm{X}$ \\
\hline 09075700 & Maroon Creek above Aspen, Colo. & $\mathrm{X}$ & $\mathrm{x}$ & $\mathrm{x}$ & $\mathrm{X}$ & $\mathrm{X}$ & $\mathrm{X}$ \\
\hline 09077200 & Fryingpan River near Ivanhoe Lake, Colo..-- & $\mathrm{X}$ & $\mathrm{x}$ & $\mathrm{X}$ & $\mathrm{X}$ & $\mathrm{X}$ & \\
\hline 09077800 & $\begin{array}{l}\text { South Fork Fryingpan River at Upper } \\
\text { Station near Norrie, Colo. }\end{array}$ & $\mathrm{x}$ & $\mathrm{x}$ & $\mathrm{X}$ & $\mathrm{X}$ & $\mathrm{x}$ & \\
\hline $\begin{array}{l}09078000 \\
09078100\end{array}$ & $\begin{array}{l}\text { Fryingpan River at Norrie, Colo. } \\
\text { North Fork Fryingpan River above }\end{array}$ & $\mathrm{x}$ & $\mathrm{x}$ & $\mathrm{x}$ & $\mathrm{x}$ & $\mathrm{x}$ & $\mathrm{X}$ \\
\hline & Cunningham Creek near Norrie, Colo. & $\mathrm{X}$ & $\mathrm{x}$ & $\mathrm{X}$ & $\mathrm{x}$ & $\mathrm{X}$ & $\mathrm{x}$ \\
\hline $\begin{array}{l}09078200 \\
09078500\end{array}$ & $\begin{array}{l}\text { Cunningham Creek near Norrie, Colo. } \\
\text { North Fork Fryingpan River near }\end{array}$ & $\mathrm{X}$ & $\mathrm{x}$ & $\mathrm{X}$ & $\mathrm{X}$ & $\mathrm{X}$ & $\mathrm{x}$ \\
\hline & Norrie, Colo. & $\mathrm{X}$ & $\mathrm{x}$ & $\mathrm{X}$ & $\mathrm{x}$ & $\mathrm{X}$ & $\mathrm{X}$ \\
\hline 09080100 & Fryingpan River at Meredith, Colo. & $\mathrm{X}$ & $\mathrm{x}$ & $\mathrm{X}$ & $\mathrm{X}$ & $\mathrm{X}$ & $\mathrm{x}$ \\
\hline 09082800 & North Thompson Creek near Carbondale, Colo.- & $\mathrm{x}$ & $\mathrm{x}$ & $\mathrm{x}$ & $\mathrm{X}$ & $\mathrm{x}$ & $\mathrm{x}$ \\
\hline $\begin{array}{l}09084000 \\
09089000\end{array}$ & $\begin{array}{l}\text { Cattle Creek near Carbondale, Colo. } \\
\text { West Divide Creek below Willow Creek, }\end{array}$ & $\mathrm{x}$ & $\mathrm{x}$ & $\mathrm{X}$ & $\mathrm{X}$ & $\mathrm{X}$ & $\mathrm{X}$ \\
\hline 09096000 & $\begin{array}{l}\text { near Raven, Colo. } \\
\text { Plateau Creek at Upper Station near }\end{array}$ & $\mathrm{X}$ & $\mathrm{x}$ & $\mathrm{X}$ & $\mathrm{X}$ & $\mathrm{X}$ & $\mathrm{X}$ \\
\hline & Collbran, Colo. & $\mathrm{X}$ & $\mathrm{X}$ & $\mathrm{X}$ & $\mathrm{x}$ & $\mathrm{X}$ & $\mathrm{X}$ \\
\hline 09096800 & Buzzard Creek below Owens Creek, near & & & & & & \\
\hline 09097600 & Brush Creek near Collbran, Colo................... & $\hat{x}$ & $\hat{X}$ & $\mathrm{X}$ & $\hat{x}$ & $\begin{array}{l}\hat{X} \\
\mathrm{X}\end{array}$ & $\begin{array}{l}\hat{X} \\
\mathrm{X}\end{array}$ \\
\hline 09110000 & Taylor River at Almont, Colo. & $\mathrm{X}$ & $\mathrm{X}$ & $\mathrm{x}$ & $\mathrm{x}$ & $\mathrm{X}$ & $\mathrm{X}$ \\
\hline 09110500 & East River near Crested Butte, Colo. & $\mathrm{X}$ & $\mathrm{X}$ & $\mathrm{X}$ & $\mathrm{X}$ & $\mathrm{X}$ & $\mathrm{X}$ \\
\hline 09111500 & Slate River near Crested Butte, Colo....... & $\mathrm{X}$ & $\mathrm{X}$ & $\mathrm{X}$ & $\mathrm{X}$ & $\mathrm{X}$ & $\mathrm{X}$ \\
\hline $\begin{array}{l}09112000 \\
09112200\end{array}$ & $\begin{array}{l}\text { Cement Creek near Crested Butte, Colo. } \\
\text { East River below Cement Creek near }\end{array}$ & $\mathrm{X}$ & $\mathrm{X}$ & $\mathbf{x}$ & $\mathrm{X}$ & $\mathrm{x}$ & $\mathrm{X}$ \\
\hline & Crested Butte, Colo. & $\mathrm{X}$ & $\mathrm{X}$ & $\mathrm{X}$ & $\mathrm{X}$ & $\mathrm{X}$ & $\mathrm{X}$ \\
\hline 09112500 & East River at Almont, Colo. & $\mathrm{X}$ & $\mathrm{x}$ & $\mathbf{X}$ & $\mathrm{X}$ & $\mathrm{X}$ & $\mathrm{X}$ \\
\hline 09113300 & Ohio Creek at Baldwin, Colo. & $\mathrm{x}$ & $\mathrm{x}$ & $\mathrm{X}$ & $\mathrm{x}$ & $\mathrm{x}$ & $\mathrm{x}$ \\
\hline 09113500 & Ohio Creek near Baldwin, Colo..... & $\mathrm{x}$ & $\mathrm{X}$ & $\mathrm{X}$ & $\mathrm{x}$ & $\mathrm{X}$ & $\mathrm{X}$ \\
\hline 09114500 & Gunnison River near Gunnison, Colo. & $\mathrm{x}$ & $\mathrm{x}$ & $\mathrm{x}$ & $\mathrm{x}$ & $\mathrm{x}$ & $\mathrm{x}$ \\
\hline 09115500 & Tomichi Creek at Sargents, Colo.... & $\mathbf{X}$ & $\mathrm{x}$ & $\mathrm{X}$ & $\mathrm{X}$ & $\mathrm{X}$ & $\mathrm{X}$ \\
\hline 09117000 & Tomichi Creek at Parlin, Colo. & $\mathrm{x}$ & $\mathrm{x}$ & $\mathrm{x}$ & $\mathrm{x}$ & $\mathrm{X}$ & $\mathrm{X}$ \\
\hline 09118000 & Quartz Creek near Ohio City, Colo. & $\mathrm{X}$ & $\mathrm{x}$ & $\mathrm{X}$ & $\mathrm{X}$ & $\mathrm{X}$ & $\mathbf{X}$ \\
\hline 09119000 & Tomichi Creek at Gunnison, Colo................. & $\mathrm{X}$ & $\mathrm{x}$ & $\mathrm{X}$ & $\mathrm{X}$ & $\mathrm{X}$ & $\mathrm{x}$ \\
\hline 09122000 & Cebolla Creek at Powderhorn, Colo. & $\mathrm{x}$ & $\mathrm{x}$ & $\mathrm{X}$ & $\mathrm{x}$ & $\mathrm{X}$ & $\mathrm{x}$ \\
\hline 09122500 & Soap Creek near Sapinero, Colo. & $\mathrm{X}$ & $\mathrm{x}$ & $\mathrm{x}$ & $\mathrm{x}$ & $\mathrm{x}$ & $\mathrm{x}$ \\
\hline 09123500 & Lake Fork at Lake City, Colo. & $\mathrm{X}$ & $\mathrm{x}$ & $\mathrm{X}$ & $\mathrm{x}$ & $\mathrm{x}$ & $\mathrm{X}$ \\
\hline 09124500 & Lake Fork at Gateview, Colo. & $\mathrm{X}$ & $\mathrm{x}$ & $\mathrm{X}$ & $\mathrm{x}$ & $\mathrm{x}$ & $\mathrm{X}$ \\
\hline 09125000 & Curecanti Creek near Sapinero, Colo. & $\mathrm{X}$ & $\mathrm{x}$ & $\mathrm{X}$ & $\mathrm{X}$ & $\mathrm{X}$ & $\mathrm{X}$ \\
\hline 09127500 & Crystal Creek near Maher, Colo. & $\mathrm{x}$ & $\mathrm{x}$ & $\mathrm{x}$ & $\mathrm{x}$ & $\mathrm{x}$ & $\mathrm{x}$ \\
\hline 09130600 & West Muddy Creek near Ragged Mountain, Colo. & $\mathrm{x}$ & $\mathrm{x}$ & $\mathrm{X}$ & $\mathrm{X}$ & $\mathrm{X}$ & $\mathrm{X}$ \\
\hline 09132900 & West Hubbard Creek near Paonia, Colo. & $\mathrm{x}$ & $\mathrm{X}$ & $\mathrm{x}$ & $\mathrm{X}$ & $\mathrm{X}$ & $\mathrm{X}$ \\
\hline 09139200 & Ward Creek near Grand Mesa, Colo................. & $\mathrm{x}$ & $\mathrm{x}$ & $\mathrm{x}$ & $\mathrm{X}$ & $\mathrm{X}$ & $\mathrm{X}$ \\
\hline 09140200 & Kiser Creek near Grand Mesa, Colo................ & $\mathrm{X}$ & $\mathrm{X}$ & $\mathrm{X}$ & $\mathrm{X}$ & $\mathrm{X}$ & $\mathrm{x}$ \\
\hline 09143000 & Surface Creek near Cedaredge, Colo..... & $\mathrm{x}$ & $\mathrm{x}$ & $\mathrm{x}$ & $\mathrm{x}$ & $\mathbf{x}$ & $\mathrm{X}$ \\
\hline 09147100 & Cow Creek near Ridgway, Colo. & $\mathrm{X}$ & $\mathrm{X}$ & $\mathrm{X}$ & $X$ & $\mathrm{X}$ & $\mathrm{X}$ \\
\hline 09244500 & Elkhead Creek near Clark, Colo. & $\mathrm{x}$ & $\mathrm{x}$ & $\mathrm{X}$ & $\mathrm{X}$ & $\mathrm{X}$ & $\mathrm{X}$ \\
\hline 09302450 & Lost Creek near Buford, Colo. & $\mathrm{x}$ & $\mathrm{x}$ & $\mathrm{X}$ & $\mathbf{X}$ & $\mathrm{X}$ & $\mathrm{X}$ \\
\hline
\end{tabular}


Table 2.--Gaging-station records used to determine regression relations for the Rio Grande region [X, gaging station was used in the analysis]

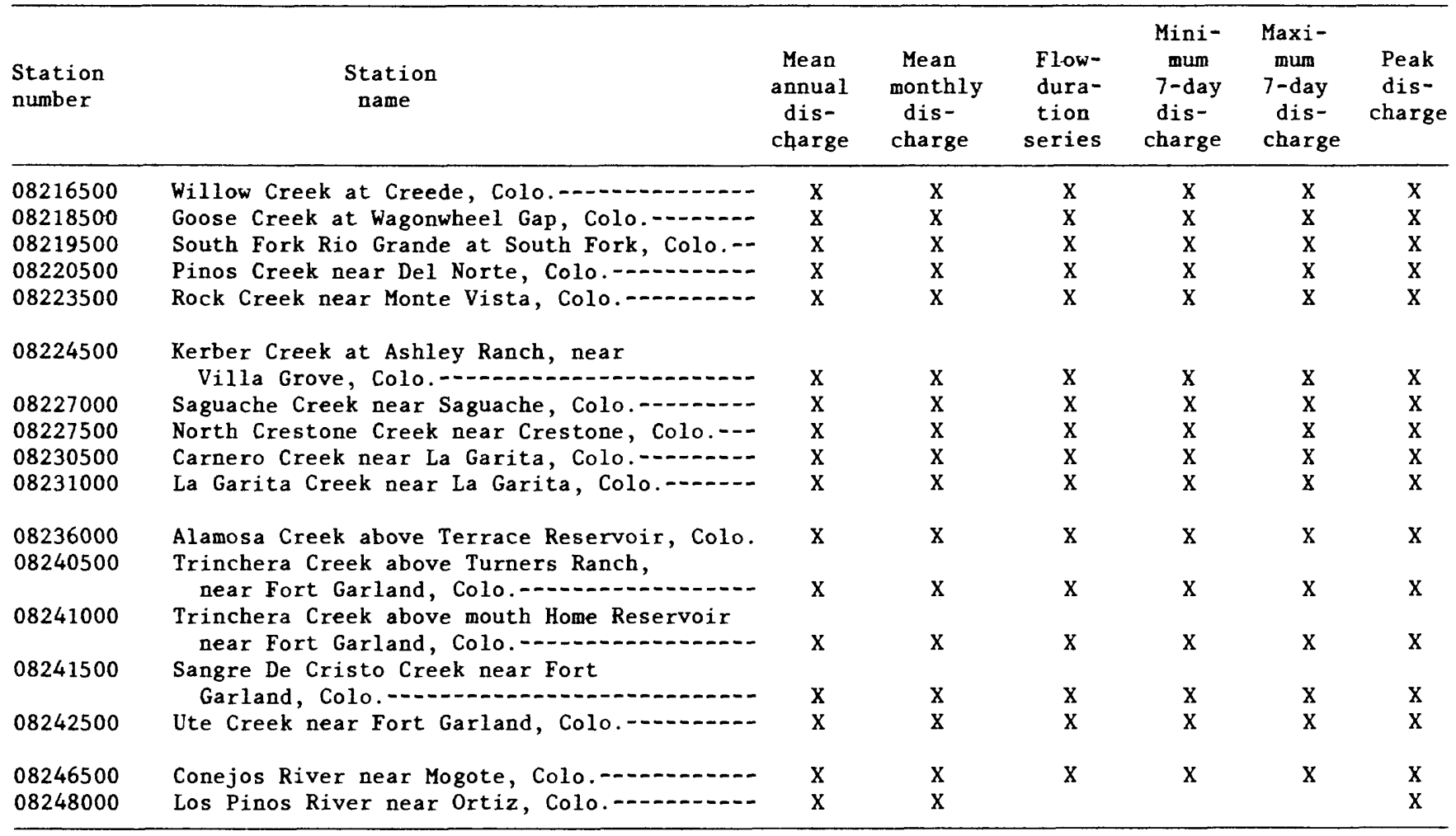


Table 3.--Gaging-station records used to determine regression relations for the southwest region [ $X$, gaging station was used in the analysis]

\begin{tabular}{|c|c|c|c|c|c|c|c|}
\hline $\begin{array}{l}\text { Station } \\
\text { number }\end{array}$ & $\begin{array}{l}\text { Station } \\
\text { name }\end{array}$ & $\begin{array}{l}\text { Mean } \\
\text { annual } \\
\text { dis- } \\
\text { charge }\end{array}$ & $\begin{array}{l}\text { Mean } \\
\text { monthly } \\
\text { dis- } \\
\text { charge }\end{array}$ & $\begin{array}{l}\text { Flow- } \\
\text { dura- } \\
\text { tion } \\
\text { series }\end{array}$ & $\begin{array}{c}\text { Mini- } \\
\text { mum } \\
\text { 7-day } \\
\text { dis- } \\
\text { charge }\end{array}$ & $\begin{array}{c}\text { Maxi- } \\
\text { mum } \\
\text { 7-day } \\
\text { dis- } \\
\text { charge }\end{array}$ & $\begin{array}{c}\text { Peak } \\
\text { dis- } \\
\text { charge }\end{array}$ \\
\hline 09145000 & Uncompahgre River at Ouray, Colo. & $\mathrm{X}$ & $\mathrm{X}$ & $\mathrm{x}$ & $\mathrm{X}$ & $\mathbf{x}$ & $\mathrm{x}$ \\
\hline 09146000 & Uncompahgre River below Ouray, Colo. & $\mathrm{X}$ & $\mathrm{x}$ & $\mathrm{x}$ & $\mathbf{x}$ & $\mathrm{x}$ & $\mathrm{x}$ \\
\hline 09146400 & West Fork Dallas Creek near Ridgway, Colo.-- & $\mathrm{X}$ & $\mathrm{x}$ & $\mathrm{x}$ & $\mathrm{x}$ & $\mathrm{x}$ & $\mathrm{x}$ \\
\hline 09146500 & East Fork Dallas Creek near Ridgway, Colo.-- & $\mathrm{X}$ & $\mathrm{X}$ & $\mathrm{x}$ & $\mathbf{X}$ & $\mathbf{x}$ & $\mathrm{x}$ \\
\hline 09146600 & Pleasant Valley Creek near Noel, Colo...- & $\mathrm{X}$ & $\mathrm{X}$ & $\mathrm{x}$ & $\mathrm{X}$ & $\mathrm{x}$ & $\mathrm{x}$ \\
\hline 09165000 & Dolores River below Rico, Colo. & $\mathbf{X}$ & $\mathrm{X}$ & $\mathrm{x}$ & $\mathrm{X}$ & $\mathrm{x}$ & $\mathrm{X}$ \\
\hline 09166500 & Dolores River at Dolores, Colo. & $\mathrm{x}$ & $\mathrm{X}$ & $\mathrm{x}$ & $\mathrm{x}$ & $\mathrm{x}$ & $\mathrm{x}$ \\
\hline 09167500 & Dolores River near McPhee, Colo. & $\mathrm{X}$ & $\mathrm{X}$ & $\mathrm{x}$ & $\mathrm{x}$ & $\mathrm{x}$ & $\mathrm{x}$ \\
\hline 09168100 & Disappointment Creek near Dove Creek, Colo.- & $\mathrm{X}$ & $\mathrm{x}$ & $\mathrm{x}$ & $\mathrm{x}$ & $\mathrm{X}$ & $\mathbf{x}$ \\
\hline 09169500 & Dolores River at Bedrock, Colo..- & $\mathrm{X}$ & $\mathrm{X}$ & $\mathrm{x}$ & $\mathrm{x}$ & $\mathrm{X}$ & $\mathrm{x}$ \\
\hline 09171100 & Dolores River near Bedrock, Colo. & $\mathrm{x}$ & $\mathrm{x}$ & $\mathrm{x}$ & $\mathbf{x}$ & $\mathrm{x}$ & $\mathbf{x}$ \\
\hline 09172500 & San Miguel River near Placerville, Colo.---- & $\mathrm{x}$ & $\mathrm{X}$ & $\mathrm{x}$ & $\mathrm{x}$ & $\mathrm{x}$ & $\mathrm{x}$ \\
\hline 09175500 & San Miguel River at Naturita, Colo..-- & $\mathrm{X}$ & $\mathrm{X}$ & $\mathrm{x}$ & $\mathbf{X}$ & $\mathrm{X}$ & $\mathrm{X}$ \\
\hline 09177000 & San Miguel River at Uravan, Colo. & $\mathrm{x}$ & $\mathrm{X}$ & $\mathrm{x}$ & $\mathrm{x}$ & $\mathrm{x}$ & $\mathrm{x}$ \\
\hline 09179500 & Dolores River at Gateway, Colo. & $\mathrm{x}$ & $\mathrm{X}$ & $\mathrm{x}$ & $\mathrm{x}$ & $\mathrm{X}$ & $\mathrm{x}$ \\
\hline 09339900 & $\begin{array}{l}\text { East Fork San Juan River above Sand Creek, } \\
\text { near Pagosa Springs, Colo. }\end{array}$ & $\mathrm{x}$ & $\mathrm{x}$ & $\mathrm{X}$ & $\mathrm{X}$ & $\mathbf{X}$ & $\mathrm{X}$ \\
\hline 09340000 & East Fork San Juan River near Pagosa & & & & & & \\
\hline & Springs, Colo..... & $\mathrm{x}$ & $\mathrm{x}$ & $\mathrm{x}$ & $\mathrm{x}$ & $\mathrm{x}$ & $\mathrm{X}$ \\
\hline 09340500 & West Fork San Juan River above Barns & & & & & & \\
\hline & Lake near Pagosa Springs, Colo. & $\mathrm{x}$ & $\mathrm{X}$ & $\mathrm{x}$ & $\mathrm{X}$ & $\mathbf{X}$ & $\mathrm{X}$ \\
\hline 09341500 & West Fork San Juan River near Pagosa & & & & & & \\
\hline & Springs, Colo. & $\mathrm{x}$ & $\mathrm{X}$ & $\mathrm{X}$ & $\mathrm{x}$ & $\mathrm{X}$ & $\mathrm{x}$ \\
\hline 09342000 & Turkey Creek near Pagosa Springs, Colo..-- & $\mathrm{x}$ & $\mathrm{X}$ & $\mathrm{X}$ & $\mathrm{x}$ & $\mathrm{X}$ & $\mathrm{X}$ \\
\hline 09342500 & San Juan River at Pagosa Springs, Colo.---- & $\mathrm{x}$ & $\mathrm{X}$ & $\mathrm{X}$ & $\mathrm{x}$ & $\mathrm{X}$ & $\mathrm{x}$ \\
\hline 09343000 & Rio Blanco near Pagosa Springs, Colo.....- & $\mathrm{X}$ & $\mathrm{X}$ & $\mathrm{X}$ & $\mathrm{x}$ & $\mathrm{X}$ & $\mathrm{X}$ \\
\hline 09343300 & Rio Blanco below Blanco Diversion Dam, & & & & & & \\
\hline & near Pagosa Springs, Colo. & $\mathrm{X}$ & $\mathrm{X}$ & $\mathrm{X}$ & $\mathrm{X}$ & $\mathrm{X}$ & \\
\hline $\begin{array}{l}09343500 \\
09344000\end{array}$ & $\begin{array}{l}\text { Rio Blanco near Pagosa Springs, Colo. } \\
\text { Navajo River at Banded Peak Ranch, }\end{array}$ & $\mathrm{X}$ & $\mathrm{X}$ & $\mathrm{X}$ & $\mathrm{x}$ & $\mathrm{x}$ & $\mathrm{X}$ \\
\hline & near Chromo, Colo. & $\mathrm{X}$ & $\mathrm{x}$ & $\mathrm{X}$ & $\mathrm{X}$ & $\mathrm{X}$ & $\mathrm{x}$ \\
\hline 09344300 & Navajo River above Chromo, Colo. & $\mathrm{X}$ & $\mathrm{x}$ & $\mathrm{x}$ & $\mathrm{x}$ & $\mathrm{x}$ & $\mathrm{X}$ \\
\hline 09344400 & $\begin{array}{l}\text { Navajo River below Oso Diversion Dam, } \\
\text { near Chromo, Colo. }\end{array}$ & $\mathrm{x}$ & $\mathrm{X}$ & $\mathrm{X}$ & $\mathrm{x}$ & $\mathrm{X}$ & \\
\hline 09345200 & Little Navajo River below Lake Oso & & & & & & \\
\hline & Diversion Dam, near Chromo, Colo. & $\mathrm{X}$ & $\mathrm{X}$ & $\mathrm{X}$ & $\mathrm{x}$ & $\mathrm{X}$ & \\
\hline 09345500 & Little Navajo River at Chromo, Colo...... & $\mathrm{X}$ & $\mathrm{X}$ & $\mathrm{X}$ & $\mathrm{x}$ & $\mathrm{X}$ & $\mathrm{x}$ \\
\hline 09346000 & Navajo River at Edith, Colo. & $\mathrm{X}$ & $\mathbf{x}$ & $\mathrm{X}$ & $\mathrm{X}$ & $\mathrm{X}$ & $\mathrm{X}$ \\
\hline 09346400 & San Juan River near Carracas, Colo..- & $\mathbf{X}$ & $\mathrm{X}$ & $\mathrm{X}$ & $\mathrm{X}$ & $\mathrm{X}$ & $\mathbf{X}$ \\
\hline 09347500 & Piedra River at Bridge Ranger Station & & & & & & \\
\hline & near Pagosa Springs, Colo. & $\mathrm{X}$ & $\mathrm{X}$ & $\mathrm{X}$ & $\mathrm{x}$ & $\mathrm{x}$ & $\mathrm{X}$ \\
\hline 09349500 & Piedra River near Piedra, Colo. & $\mathrm{X}$ & $\mathrm{x}$ & $\mathrm{X}$ & $\mathrm{x}$ & $\mathrm{X}$ & $\mathrm{X}$ \\
\hline 09349800 & Piedra River near Arboles, Colo. & $\mathrm{x}$ & $\mathrm{x}$ & $\mathrm{X}$ & $\mathbf{x}$ & $\mathrm{x}$ & $\mathbf{X}$ \\
\hline 09350500 & San Juan River at Rosa, N. Mex.- & $\mathrm{x}$ & $\mathrm{x}$ & $\mathrm{x}$ & $\mathrm{X}$ & $\mathrm{X}$ & $\mathrm{x}$ \\
\hline 09352900 & Vallecito Creek near Bayfield, Colo. & $\mathrm{X}$ & $\mathrm{x}$ & $\mathrm{X}$ & $\mathrm{x}$ & $\mathrm{X}$ & $\mathrm{x}$ \\
\hline 09353500 & Los Pinos River near Bayfield, Colo. & $\mathrm{X}$ & $\mathrm{x}$ & $x$ & $\mathrm{x}$ & $\mathrm{x}$ & $\mathrm{X}$ \\
\hline 09355000 & Spring Creek at La Boca, Colo. & $\mathrm{X}$ & $\mathrm{X}$ & $\mathrm{X}$ & $\mathrm{x}$ & $\mathrm{x}$ & $\mathrm{x}$ \\
\hline 09357500 & Animas River at Howardsville, Colo..... & $\mathrm{X}$ & $\mathrm{X}$ & $\mathrm{X}$ & $\mathrm{X}$ & $\mathrm{x}$ & $\mathrm{x}$ \\
\hline 09359000 & Mineral Creek near Silverton, Colo.-- & $\mathrm{X}$ & $\mathrm{X}$ & $\mathrm{X}$ & $\mathrm{X}$ & $\mathrm{X}$ & $X$ \\
\hline
\end{tabular}


Table 3.--Gaging-station records used to determine regression relations for the southwest region--Continued

\begin{tabular}{|c|c|c|c|c|c|c|c|}
\hline $\begin{array}{l}\text { Station } \\
\text { number }\end{array}$ & $\begin{array}{l}\text { Station } \\
\text { name }\end{array}$ & $\begin{array}{c}\text { Mean } \\
\text { annual } \\
\text { dis- } \\
\text { charge }\end{array}$ & $\begin{array}{l}\text { Mean } \\
\text { monthly } \\
\text { dis- } \\
\text { charge }\end{array}$ & $\begin{array}{l}\text { Flow- } \\
\text { dura- } \\
\text { tion } \\
\text { series }\end{array}$ & $\begin{array}{c}\text { Mini- } \\
\text { mum } \\
7 \text {-day } \\
\text { dis- } \\
\text { charge }\end{array}$ & $\begin{array}{l}\text { Maxi- } \\
\text { mum } \\
\text { 7-day } \\
\text { dis- } \\
\text { charge }\end{array}$ & $\begin{array}{c}\text { Peak } \\
\text { dis- } \\
\text { charge }\end{array}$ \\
\hline 09359500 & Animas River above Tacoma, Colo, & $\mathrm{X}$ & $\mathrm{x}$ & $\mathrm{x}$ & $\mathrm{X}$ & $\mathrm{X}$ & $\mathrm{X}$ \\
\hline 09361000 & Hermosa Creek near Hermosa, Colo. & $\mathrm{X}$ & $\mathrm{x}$ & $\mathrm{X}$ & $\mathrm{X}$ & $\mathrm{x}$ & $\mathrm{X}$ \\
\hline 09361500 & Animas River at Durango, Colo. & $\mathrm{X}$ & $\mathrm{X}$ & $\mathrm{X}$ & $\mathrm{X}$ & $\mathrm{x}$ & $\mathrm{X}$ \\
\hline 09362000 & Lightner Creek near Durango, Colo. & $\mathrm{X}$ & $\mathrm{X}$ & $\mathrm{X}$ & $\mathrm{X}$ & $\mathbf{X}$ & $\mathrm{X}$ \\
\hline 09363000 & Florida River near Durango, Colo. & $\mathrm{X}$ & $\mathrm{X}$ & $\mathrm{X}$ & $\mathrm{X}$ & $\mathrm{X}$ & $\mathbf{x}$ \\
\hline \multirow{5}{*}{$\begin{array}{l}09363100 \\
09363500 \\
09365500 \\
09366000 \\
09366500\end{array}$} & Salt Creek near Oxford, Colo. & $\mathrm{X}$ & $\mathrm{X}$ & $\mathrm{X}$ & $\mathrm{X}$ & $\mathrm{X}$ & $\mathrm{X}$ \\
\hline & Animas River near Cedar Hill, N. Mex......... & $\mathrm{X}$ & $\mathrm{X}$ & $\mathrm{X}$ & $\mathrm{X}$ & $\mathrm{X}$ & $\mathrm{X}$ \\
\hline & La Plata River at Hesperus, Colo. & $\mathrm{X}$ & $\mathrm{X}$ & $\mathrm{X}$ & $\mathrm{X}$ & $\mathrm{X}$ & $\mathrm{X}$ \\
\hline & Cherry Creek near Red Mesa, Colo. & $\mathrm{X}$ & $\mathrm{X}$ & $\mathrm{X}$ & $\mathrm{X}$ & $\mathrm{X}$ & $\mathrm{X}$ \\
\hline & $\begin{array}{l}\text { La Plata River at the Colorado-New Mexico } \\
\text { State line- }\end{array}$ & $\mathrm{X}$ & $\mathrm{X}$ & $\mathrm{X}$ & $\mathrm{X}$ & $\mathrm{X}$ & $\mathrm{X}$ \\
\hline 09369000 & East Mancos River near Mancos, Colo..... & $\mathrm{X}$ & $\mathrm{X}$ & $\mathrm{x}$ & $\mathrm{X}$ & $\mathrm{X}$ & $\mathrm{X}$ \\
\hline 09369500 & Middle Mancos River near Mancos, Colo.....- & $\mathrm{X}$ & $\mathrm{X}$ & $\mathrm{X}$ & $\mathrm{X}$ & $\mathrm{X}$ & $\mathrm{X}$ \\
\hline 09371500 & 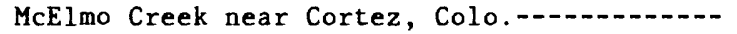 & $\mathrm{X}$ & $\mathrm{X}$ & $\mathrm{X}$ & $\mathrm{X}$ & $\mathrm{X}$ & $\mathrm{X}$ \\
\hline 09372000 & McElmo Creek near Colo-Utah State line---.- & $\mathrm{X}$ & $\mathrm{X}$ & $\mathrm{X}$ & $\mathrm{X}$ & $\mathrm{X}$ & $\mathbf{X}$ \\
\hline
\end{tabular}


Table 4.--Gaging-station records used to determine regression relations for the northwest region [X, gaging station was used in the analysis]

\begin{tabular}{|c|c|c|c|c|c|c|c|}
\hline $\begin{array}{l}\text { Station } \\
\text { number }\end{array}$ & $\begin{array}{l}\text { Station } \\
\text { name }\end{array}$ & $\begin{array}{l}\text { Mean } \\
\text { annual } \\
\text { dis- } \\
\text { charge }\end{array}$ & $\begin{array}{c}\text { Mean } \\
\text { monthly } \\
\text { dis- } \\
\text { charge }\end{array}$ & $\begin{array}{l}\text { Flow- } \\
\text { dura- } \\
\text { tion } \\
\text { series }\end{array}$ & $\begin{array}{l}\text { Mini- } \\
\text { mum } \\
7 \text {-day } \\
\text { dis- } \\
\text { charge }\end{array}$ & $\begin{array}{l}\text { Maxi- } \\
\text { mum } \\
7 \text {-day } \\
\text { dis- } \\
\text { charge }\end{array}$ & $\begin{array}{c}\text { Peak } \\
\text { dis- } \\
\text { charge }\end{array}$ \\
\hline 09040500 & Troublesome Creek near Troublesome, Colo.--- & $\mathrm{X}$ & $\mathrm{X}$ & $\mathrm{X}$ & $\mathrm{X}$ & $\mathrm{X}$ & $\mathrm{X}$ \\
\hline 09059500 & Piney River near State Bridge, Colo.-- & $\mathrm{X}$ & $\mathrm{X}$ & $\mathrm{X}$ & $\mathrm{X}$ & $\mathrm{X}$ & $\mathrm{x}$ \\
\hline 09067500 & Eagle River at Eagle, Colo. & $\mathrm{X}$ & $\mathrm{X}$ & $\mathrm{X}$ & $\mathrm{X}$ & $\mathrm{X}$ & $\mathrm{X}$ \\
\hline 09068000 & 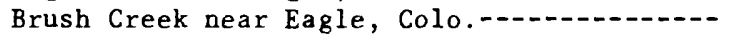 & $\mathrm{X}$ & $\mathrm{X}$ & $\mathrm{X}$ & $\mathrm{X}$ & $\mathrm{X}$ & $\mathrm{X}$ \\
\hline 09069000 & Eagle River at Gypsum, Colo... & $\mathrm{X}$ & $\mathrm{X}$ & $\mathrm{X}$ & $\mathrm{X}$ & $\mathrm{X}$ & $\mathrm{X}$ \\
\hline 09070000 & Eagle River below Gypsum, Colo..... & $\mathrm{X}$ & $\mathrm{X}$ & $\mathrm{X}$ & $\mathrm{X}$ & $\mathrm{X}$ & $\mathrm{X}$ \\
\hline 09080300 & Rocky Fork Creek near Meredith, Colo.....- & $\mathrm{X}$ & $\mathbf{X}$ & $\mathrm{X}$ & $\mathrm{X}$ & $\mathrm{X}$ & $\mathrm{X}$ \\
\hline 09080400 & 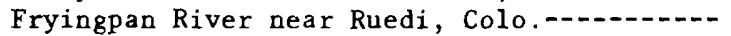 & $\mathrm{X}$ & $\mathrm{X}$ & $\mathrm{X}$ & $\mathrm{X}$ & $\mathrm{X}$ & \\
\hline \multirow[t]{2}{*}{$\begin{array}{l}09081550 \\
09081600\end{array}$} & $\begin{array}{l}\text { Crystal River at Placita, Colo. } \\
\text { Crystal River above Avalanche Creek }\end{array}$ & $\mathrm{X}$ & $\mathrm{X}$ & $\mathrm{X}$ & $\mathrm{X}$ & $\mathrm{X}$ & $\mathrm{X}$ \\
\hline & near Redstone, Colo. & $\mathrm{X}$ & $\mathrm{X}$ & $\mathrm{X}$ & $\mathbf{X}$ & $\mathrm{X}$ & $\mathrm{X}$ \\
\hline 09082500 & Crystal River near Redstone, Colo..- & $\mathrm{X}$ & $\mathrm{X}$ & $\mathrm{X}$ & $\mathrm{X}$ & $\mathrm{X}$ & $\mathrm{X}$ \\
\hline 09083000 & Thompson Creek near Carbondale, Colo..... & $\mathrm{X}$ & $\mathrm{X}$ & $\mathrm{X}$ & $\mathrm{X}$ & $\mathrm{X}$ & $\mathrm{X}$ \\
\hline 09085200 & Canyon Creek above New Castle, Colo..... & $\mathrm{X}$ & $\mathrm{X}$ & $\mathrm{X}$ & $\mathrm{X}$ & $\mathrm{X}$ & $\mathrm{X}$ \\
\hline 09085300 & East Canyon Creek near New Castle, Colo..-- & $\mathrm{X}$ & $\mathrm{X}$ & $\mathbf{X}$ & $\mathrm{X}$ & $\mathrm{X}$ & $\mathrm{X}$ \\
\hline 09085400 & Possum Creek near New Castle, Colo:-.......... & $\mathrm{X}$ & $\mathrm{X}$ & $\mathrm{X}$ & $\mathrm{X}$ & $\mathrm{X}$ & $\mathrm{X}$ \\
\hline 09089500 & West Divide Creek near Raven, Colo.....- & $\mathrm{X}$ & $\mathrm{X}$ & $\mathrm{X}$ & $\mathbf{X}$ & $\mathrm{X}$ & $\mathrm{X}$ \\
\hline 09091500 & 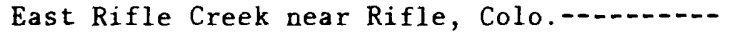 & $\mathrm{X}$ & $\mathrm{X}$ & $\mathrm{X}$ & $\mathrm{X}$ & $\mathrm{X}$ & \\
\hline 09092000 & Rifle Creek near Rifle, Colo. & $\mathrm{X}$ & $\mathrm{X}$ & $\mathrm{X}$ & $\mathrm{X}$ & $\mathrm{X}$ & $\mathrm{X}$ \\
\hline 09092500 & Beaver Creek near Rifle, Colo... & $\mathrm{X}$ & $\mathrm{X}$ & $\mathrm{X}$ & $\mathrm{X}$ & $\mathrm{X}$ & $\mathrm{X}$ \\
\hline 09093000 & Parachute Creek near Parachute, Colo..-.- & $\mathrm{X}$ & $\mathrm{X}$ & $\mathrm{X}$ & $\mathrm{X}$ & $\mathrm{X}$ & $\mathrm{X}$ \\
\hline 09093500 & Parachute Creek at Parachute, Colo..... & $\mathrm{X}$ & $\mathrm{X}$ & $\mathrm{X}$ & $\mathrm{X}$ & $\mathrm{X}$ & $\mathrm{X}$ \\
\hline 09095000 & Roan Creek near De Beque, Colo. & $\mathrm{X}$ & $\mathrm{X}$ & $\mathbf{X}$ & $\mathrm{X}$ & $\mathrm{X}$ & $\mathrm{X}$ \\
\hline 09097500 & 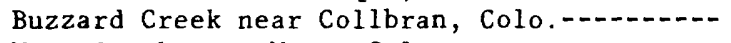 & $\mathrm{X}$ & $\mathrm{X}$ & $\mathrm{X}$ & $\mathrm{X}$ & $\mathrm{X}$ & $\mathrm{X}$ \\
\hline 09104500 & Mesa Creek near Mesa, Colo. & $\mathrm{X}$ & $\mathrm{X}$ & $\mathrm{X}$ & $\mathrm{X}$ & $\mathrm{X}$ & $\mathrm{X}$ \\
\hline 09123000 & Soap Creek at Sapinero, Colo. & $\mathrm{X}$ & $\mathrm{X}$ & $\mathrm{X}$ & $\mathrm{X}$ & $\mathrm{X}$ & $\mathrm{X}$ \\
\hline 09128500 & Smith Fork near Crawford, Colo. & $\mathrm{X}$ & $\mathrm{X}$ & $\mathrm{X}$ & $\mathrm{X}$ & $\mathrm{X}$ & $\mathrm{X}$ \\
\hline \multirow{3}{*}{$\begin{array}{l}09130500 \\
09132500\end{array}$} & East Muddy Creek near Bardine, Colo. & $\mathrm{X}$ & $\mathrm{X}$ & $\mathrm{X}$ & $\mathrm{X}$ & $\mathrm{X}$ & $\mathrm{X}$ \\
\hline & North Fork Gunnison River near & & & & & & \\
\hline & Somerset, Colo. & $\mathrm{X}$ & $\mathrm{X}$ & $\mathrm{X}$ & $\mathrm{X}$ & $\mathrm{X}$ & $X$ \\
\hline 09134500 & Leroux Creek near Cedaredge, Colo..... & $\mathrm{X}$ & $\mathrm{X}$ & $\mathrm{X}$ & $\mathrm{X}$ & $\mathrm{X}$ & $\mathrm{X}$ \\
\hline 09136200 & Gunnison River near Lazear, Colo.............. & $\mathrm{X}$ & $\mathrm{X}$ & $\mathrm{X}$ & $\mathbf{X}$ & $\mathrm{X}$ & $\mathrm{X}$ \\
\hline 09137800 & Dirty George Creek near Grand Mesa, Colo..-- & $\mathrm{X}$ & $\mathrm{X}$ & $\mathrm{X}$ & $\mathrm{X}$ & $\mathrm{X}$ & $\mathrm{X}$ \\
\hline 09141200 & Youngs Creek near Grand Mesa, Colo............ & $\mathrm{X}$ & $\mathrm{X}$ & $\mathrm{X}$ & $\mathrm{X}$ & $\mathrm{X}$ & $\mathrm{X}$ \\
\hline 09143500 & Surface Creek at Cedaredge, Colo..... & $\mathrm{X}$ & $\mathrm{X}$ & $\mathrm{X}$ & $\mathrm{X}$ & $\mathrm{X}$ & $\mathrm{X}$ \\
\hline 09144200 & Tongue Creek at Cory, Colo. & $\mathrm{X}$ & $\mathrm{X}$ & $\mathrm{X}$ & $\mathrm{X}$ & $\mathrm{X}$ & $\mathrm{X}$ \\
\hline 09146200 & Uncompahgre River near Ridgway, Colo..... & $\mathrm{X}$ & $\mathrm{X}$ & $\mathrm{X}$ & $\mathrm{X}$ & $\mathrm{X}$ & $\mathrm{X}$ \\
\hline 09147000 & Dallas Creek near Ridgway, Colo.... & $\mathrm{X}$ & $\mathrm{X}$ & $\mathrm{X}$ & $\mathrm{X}$ & $\mathrm{X}$ & $X$ \\
\hline 09147500 & Uncompahgre River at Colona, Colo.... & $\mathrm{X}$ & $\mathrm{X}$ & $\mathrm{X}$ & $\mathbf{X}$ & $\mathrm{X}$ & $\mathrm{X}$ \\
\hline 09150500 & Roubideau Creek at mouth, near Delta, Colo.- & $\mathrm{X}$ & $\mathrm{X}$ & $\mathrm{X}$ & $\mathrm{X}$ & $\mathbf{X}$ & $\mathrm{X}$ \\
\hline 09239500 & Yampa River at Steamboat Springs, Colo, & $\mathrm{X}$ & $\mathrm{X}$ & $\mathrm{X}$ & $\mathrm{X}$ & $\mathrm{X}$ & $\mathrm{X}$ \\
\hline 09242500 & Elk River near Trull, Colo. & $\mathrm{X}$ & $\mathrm{X}$ & $\mathrm{X}$ & $\mathrm{X}$ & $\mathrm{X}$ & $\mathrm{X}$ \\
\hline 09244100 & Fish Creek near Milner, Colo. & $\mathrm{X}$ & $\mathrm{X}$ & $\mathrm{X}$ & $\mathrm{X}$ & $\mathrm{X}$ & $\mathrm{X}$ \\
\hline \multirow[t]{2}{*}{09244410} & Yampa River below Diversion, near & & & & & & \\
\hline & Hayden, Colo, & $\mathrm{X}$ & $\mathrm{X}$ & $\mathrm{X}$ & $\mathrm{X}$ & $\mathrm{X}$ & $\mathrm{X}$ \\
\hline 09245000 & Elkhead Creek near Elkhead, Colo. & $\mathrm{X}$ & $\mathrm{X}$ & $\mathrm{X}$ & $\mathrm{X}$ & $\mathrm{X}$ & $\mathrm{X}$ \\
\hline \multirow[t]{2}{*}{09245500} & North Fork Elkhead Creek near & & & & & & \\
\hline & Elkhead, Colo. & $\mathrm{X}$ & $\mathrm{X}$ & $\mathrm{X}$ & $\mathrm{X}$ & $\mathrm{X}$ & $\mathrm{X}$ \\
\hline 09247000 & Fortification Creek at Craig, Colo. & $X$ & $\mathrm{X}$ & $\mathrm{X}$ & & $\mathrm{X}$ & $\mathrm{X}$ \\
\hline
\end{tabular}


Table 4.--Gaging-station records used to determine regression relations for the northwest region--Continued

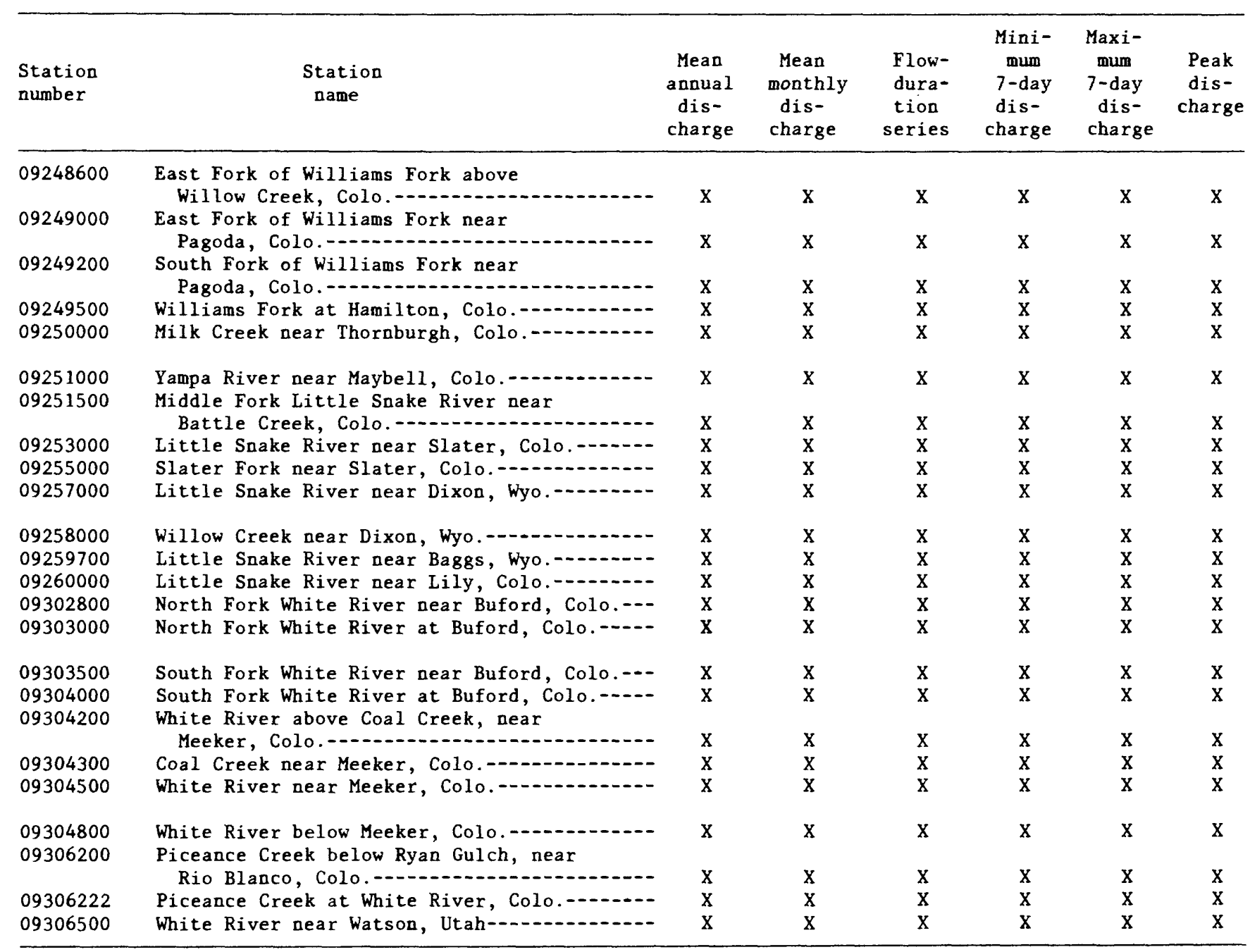


selected flow characteristics using data from all of the gaging stations. These relations included four independent variables selected from the previously defined set of basin and climatic characteristics and were defined using stepwise regression procedures (explained in the following section titled, "Regression Relations"). Residuals, which are the differences between the logs of measured and estimated flow values, then were plotted on a location map of the gaging stations. Boundaries were drawn around physiographic regions in which the regression relations tended to overestimate or underestimate streamflow.

After these initial regions were defined, regression relations were determined independently for each region. Techniques used to select variables for the final regression relations are described in detail later in this report. The regions then were reevaluated on the basis of areal plots of the residuals obtained from these refined models. If consistent deviations occurred in specific areas of a hydrologic region, regional boundaries were redefined, and regression relations were then redetermined. This procedure was repeated until residuals failed to show systematic areal distributions, and further subdivision failed to improve the precision of the estimates from the regression relation.

Four hydrologic regions were delineated in the study area (p1. 1), based on relations between streamflow and basin characteristics. These regions are subsequently referred to as the mountain region, Rio Grande region, southwest region, and northwest region.

The mountain region consists predominantly of the high peaks of the Rocky Mountains north of the Rio Grande drainage basin. Minimum elevation in this region is 7,500 ft, and the area exhibits high topographic relief. The Rio Grande region includes the Rio Grande drainage basin and the headwaters of the Arkansas drainage basin on the eastern slope of the Sangre de Cristo Mountains. The Rio Grande region includes the eastern San Juan Mountains, the San Luis Valley, and the Sangre de Cristo Mountains; elevations range from about 7,500 to $14,000 \mathrm{ft}$. The southwest region includes an area that extends west from the Continental Divide in the San Juan Mountains and south from the Uncompahgre Plateau. Elevations in this region range from about 5,000 ft near the Colorado-Utah border to $14,000 \mathrm{ft}$ along the Continental Divide. The northwest region is located north of the Uncompahgre Plateau and west of the mountain region and is an area of comparatively low elevations. Elevations in this region range from about 5,000 to $7,500 \mathrm{ft}$, with the exception of the 8,000 to $9,000-f t$ high Roan Plateau in the central part of the region.

The boundaries between the hydrologic regions were determined initially on the basis of statistical analyses, physiography, and climate. The Continental Divide forms a topographic barrier between the mountain and Rio Grande regions and between the Rio Grande and southwest regions. The Uncompahgre Plateau, which reaches elevations of 9,000 to 10,000 ft, separates the northwest and southwest regions. The Uncompahgre Plateau forms a major orographic barrier to air masses moving from the south and southwest; annual precipitation south of the Uncompahgre Plateau, in the southwest region, ranges from about 12 to 25 in., decreasing to 8 in. at the lower elevations north of the Uncompahgre Plateau in the northwest region (Colorado Climate Center, 1984). 
The 7,500-ft elevation contour forming the boundary around the northwest region approximately follows the boundary that separates snowmelt-dominated floods in the higher elevations from rainfall-dominated floods at the lower elevations (Jarrett and Costa, 1982; Elliott and others, 1982; McCain and Jarrett, 1976).

\section{Regression Relations}

Regression relations were selected using stepwise regression procedures by the Statistical Analysis System (SAS Institute, 1982, p. 101-110) and al1 possible subsets of independent variables (SAS Institute, 1982, p. 85-90). Stepwise algorithms were used to eliminate independent variables that failed to explain a significant part of the variation of the streamflow characteristics. All possible subset and stepwise regression procedures then were performed on the remaining set of variables to determine the final models. Mallows' Cp statistic (Mallows, 1964, 1973; SAS Institute, 1982, p. 103), the coefficient of determination $\left(\mathrm{r}^{2}\right)$, the mean standard error, and ease of measurement of the independent variables were all considered in the selection of variables to include in the models.

The streamflow regression relations developed for the four regions of the study area are summarized in table 5, the mountain region; table 6 , the Rio Grande region; table 7 , the southwest region; and table 8 , the northwest region. Only the coefficients significant to the regression relations are listed. In addition to the regression relations, the tables show the number of stations used to develop each relation, and the mean standard error associated with the regression relations. Regression relations are not included for some of the low-flow characteristics due to the large mean standard errors. Methods for measuring the basin and climatic characteristics that appear in the regression relations are defined for each variable in the "Glossary."

\section{Application of Regression Relations}

The first step in determining streamflow characteristics at a site is to locate the site on plate 1 and determine if the site is gaged or ungaged; then one of several computational procedures may be used. The techniques for determining flow for each of these categories are described below.

\section{Gaged Sites}

For determining station streamflow characteristics, except for peak discharge, a mean of the value of the station's streamflow characteristic from the station's historical record (Richter and others, 1984) and an estimate from the appropriate regression relation (tables 5-8) should be used. For determining peak discharge, a weighted estimate is considered to be the best estimate of flood frequency at a gaged site on an unregulated stream. 
Table 5.--Summary of regression relations for the mountain region Model: $Q=a A\left(b_{1}\right) P^{\left(b_{2}\right)} E_{B}\left(b_{3}\right) S_{B}\left(b_{4}\right)$

[Q, discharge, in cubic feet per second; $A$, drainage area in square miles; $P$, (mean annual precipitation, in inches -10 inches); $E_{B}$, (mean basin elevation, in feet $-5,000$ feet) per 1,000 feet; $S_{B}$, mean basin slope, in feet per feet; $a, b_{1}$, $b_{2}, b_{3}, b_{4}$, regression coefficients]

\begin{tabular}{|c|c|c|c|c|c|c|c|}
\hline \multirow{2}{*}{$\begin{array}{c}\text { Discharge } \\
\text { characteristic } \\
Q\end{array}$} & \multirow{2}{*}{$\begin{array}{c}\text { Regression } \\
\text { constant } \\
\mathbf{a}\end{array}$} & \multicolumn{4}{|c|}{$\begin{array}{l}\text { Regression coefficient } \\
\text { of basin characteristics }\end{array}$} & \multirow{2}{*}{$\begin{array}{l}\text { Number } \\
\text { of } \\
\text { stations }\end{array}$} & \multirow{2}{*}{$\begin{array}{l}\text { Mean stan- } \\
\text { dard error } \\
\text { (in percent) }\end{array}$} \\
\hline & & $\mathrm{b}_{1}$ & $\mathrm{~b}_{2}$ & $\mathrm{~b}_{3}$ & $\mathrm{~b}_{4}$ & & \\
\hline Annual mean- - & $4.22 \times 10^{-2}$ & 0.852 & & 2.15 & & 123 & 43 \\
\hline October mean- & $1.64 \times 10^{-3}$ & .969 & & 3.16 & & 123 & 50 \\
\hline November mean--.-- & $2.43 \times 10^{-3}$ & 1.02 & & 2.65 & & 123 & 43 \\
\hline December mean----- & $2.05 \times 10^{-3}$ & 1.04 & & 2.55 & & 123 & 45 \\
\hline January mean------ & $1.76 \times 10^{-3}$ & 1.05 & & 2.52 & & 123 & 49 \\
\hline February mean----- & $2.65 \times 10^{-3}$ & 1.06 & & 2.24 & & 123 & 49 \\
\hline March mean---- & $1.78 \times 10^{-2}$ & 1.06 & & 1.21 & & 123 & 43 \\
\hline April mean-- & $1.27 \times 10^{-1}$ & 1.07 & .373 & & & 123 & 56 \\
\hline May mean- & $5.60 \times 10^{-1}$ & .895 & .602 & & & 123 & 58 \\
\hline June mean- & $4.74 \times 10^{-2}$ & .800 & & 3.04 & & 123 & 51 \\
\hline July mean------- & $3.94 \times 10^{-4}$ & .859 & & 5.19 & & 123 & 63 \\
\hline August mean-- & $2.01 \times 10^{-4}$ & .963 & & 4.81 & & 123 & 70 \\
\hline September mean---- & $3.96 \times 10^{-4}$ & .965 & & 4.12 & & 123 & 63 \\
\hline 90 percent duration & $5.54 \times 10^{-4}$ & 1.09 & & 2.94 & & 123 & 59 \\
\hline 70 percent duration & $1.53 \times 10^{-3}$ & 1.04 & & 2.70 & & 123 & 46 \\
\hline 50 percent duration & $2.25 \times 10^{-3}$ & 0.998 & & 2.84 & & 123 & 45 \\
\hline 25 percent duration & $6.79 \times 10^{-3}$ & 0.934 & & 2.97 & & 123 & 53 \\
\hline 10 percent duration & $1.55 \times 10^{-1}$ & .813 & & 2.11 & & 123 & 52 \\
\hline 2-year-7-day low--- & $2.77 \times 10^{-4}$ & 1.08 & & 3.31 & & 122 & 62 \\
\hline 10 -year-7-day low-- & $2.54 \times 10^{-5}$ & 1.14 & & 4.27 & & 122 & 101 \\
\hline 50-year-7-day low-- & $3.39 \times 10^{-6}$ & 1.18 & & 5.13 & & 122 & 158 \\
\hline 2-year-7-day high-- & $4.23 \times 10^{1}$ & .736 & & & .861 & 123 & 50 \\
\hline 10-year-7-day high- & $5.45 \times 10^{1}$ & .754 & & & .721 & 123 & 42 \\
\hline 50-year-7-day high- & $6.38 \times 10^{1}$ & .760 & & & .668 & 123 & 42 \\
\hline 100-year-7-day high & $6.74 \times 10^{1}$ & .761 & & & .652 & 123 & 43 \\
\hline 2-year peak --- & $7.43 \times 10^{1}$ & .693 & & & .894 & 112 & 51 \\
\hline 5-year peak-.-- & $8.15 \times 10^{1}$ & .698 & & & .719 & 112 & 46 \\
\hline 10 -year peak-.-... & $8.61 \times 10^{1}$ & .699 & & & .635 & 112 & 45 \\
\hline 25-year peak---- & $9.15 \times 10^{1}$ & .699 & & & .550 & 112 & 44 \\
\hline 50-year peak- & $9.49 \times 10^{1}$ & .699 & & & .497 & 112 & 44 \\
\hline 100 -year peak--.-- & $9.85 \times 10^{1}$ & .698 & & & .452 & 112 & 45 \\
\hline 200-year peak & $1.02 \times 10^{2}$ & .697 & & & .412 & 112 & 45 \\
\hline 500-year peak-...- & $1.06 \times 10^{2}$ & .696 & & & .364 & 112 & 46 \\
\hline
\end{tabular}


Table 6.--Summary of regression relations for the Rio Grande region Model: $Q=a^{\left(b_{1}\right)}{ }_{P}^{\left(b_{2}\right)} E_{B}\left(b_{3}\right) S_{B}\left(b_{4}\right)$

[Q, discharge, in cubic feet per second; $A$, drainage area in square miles; $P$, (mean annual precipitation, in inches - 10 inches); $E_{B}$, (mean basin elevation, in feet $-5,000$ feet) per 1,000 feet; $S_{B}$, mean basin slope, in feet per feet; $a, b_{1}$, $b_{2}, b_{3}, b_{4}$, regression coefficients]

\begin{tabular}{|c|c|c|c|c|c|c|}
\hline $\begin{array}{c}\text { Discharge } \\
\text { characteristic } \\
Q\end{array}$ & $\begin{array}{c}\text { Regression } \\
\text { constant } \\
\text { a }\end{array}$ & \multicolumn{3}{|c|}{$\begin{array}{l}\text { Regression coefficient } \\
\text { of basin characteristics }\end{array}$} & $\begin{array}{l}\text { Number } \\
\text { of } \\
\text { stations }\end{array}$ & $\begin{array}{l}\text { Mean stan- } \\
\text { dard error } \\
\text { (in percent) }\end{array}$ \\
\hline Annual mean- & $5.71 \times 10^{-3}$ & 0.821 & 1.89 & & 17 & 69 \\
\hline October mean--.-- & $6.36 \times 10^{-3}$ & .755 & 1.65 & & 17 & 61 \\
\hline November mean- & $1.02 \times 10^{-2}$ & .766 & 1.37 & & 17 & 61 \\
\hline December mean- & $7.18 \times 10^{-3}$ & .748 & 1.40 & & 17 & 56 \\
\hline January mean----- & $6.85 \times 10^{-3}$ & .775 & 1.33 & & 17 & 58 \\
\hline February mean--- & $8.70 \times 10^{-3}$ & .809 & 1.22 & & 17 & 51 \\
\hline March mean- & $1.19 \times 10^{-2}$ & .889 & 1.11 & & 17 & 42 \\
\hline April mean- & $1.34 \times 10^{-2}$ & .948 & 1.40 & & 17 & 57 \\
\hline May mean- & $2.63 \times 10^{-2}$ & .853 & 1.72 & & 17 & 69 \\
\hline June mean-- & $5.45 \times 10^{-3}$ & .792 & 2.39 & & 17 & 87 \\
\hline July mean- & $3.67 \times 10^{-3}$ & .750 & 2.24 & & 17 & 82 \\
\hline August mean & $1.31 \times 10^{-2}$ & .666 & 1.73 & & 17 & 65 \\
\hline September mean-- & $4.19 \times 10^{-3}$ & .714 & 1.93 & & 17 & 75 \\
\hline 90 percent duration & $3.39 \times 10^{-4}$ & .671 & 2.49 & & 16 & 105 \\
\hline 70 percent duration & $5.33 \times 10^{-3}$ & .750 & 1.53 & & 16 & 62 \\
\hline 50 percent duration & $7.74 \times 10^{-3}$ & .751 & 1.53 & & 16 & 59 \\
\hline 25 percent duration & $4.66 \times 10^{-3}$ & 0.774 & 2.01 & & 16 & 71 \\
\hline 10 percent duration & $7.51 \times 10^{-3}$ & .793 & 2.16 & & 16 & 80 \\
\hline 2-year-7-day low--- & $4.73 \times 10^{-4}$ & .691 & 2.31 & & 16 & 93 \\
\hline 10-year-7-day low-- & $8.20 \times 10^{-6}$ & .778 & 3.40 & & 16 & 131 \\
\hline 50-year-7-day low & $\ldots--N$ & o Usabl & e Relation & ined. & & \\
\hline 2-year-7-day high-- & $1.07 \times 10^{-2}$ & .781 & 2.27 & & 16 & 81 \\
\hline 10-year-7-day high- & $1.88 \times 10^{-1}$ & .739 & 1.59 & & 16 & 61 \\
\hline 50-year-7-day high- & 1.09 & .721 & 1.14 & & 16 & 51 \\
\hline 100 -year-7-day high & 2.05 & .715 & .976 & & 16 & 49 \\
\hline 2-year peak- & $5.04 \times 10^{-2}$ & .806 & 1.87 & & 17 & 70 \\
\hline 5-year peak- & $2.29 \times 10^{-1}$ & .777 & 1.55 & & 17 & 61 \\
\hline 10-year peak---- & $4.87 \times 10^{-1}$ & .760 & 1.40 & & 17 & 58 \\
\hline 25-year peak-- & 1.06 & .742 & 1.25 & & 17 & 57 \\
\hline 50-year peak---- & 1.75 & .730 & 1.16 & & 17 & 57 \\
\hline 100-year peak--.- & 2.71 & .719 & 1.07 & & 17 & 58 \\
\hline 200-year peak----- & 4.01 & .708 & 1.00 & & 17 & 59 \\
\hline 500-year peak-.-- & 6.40 & .695 & .918 & & 17 & 63 \\
\hline
\end{tabular}


Table 7.--Summary of regression relations for the southwest region

$$
\text { Model: } Q=a A\left(b_{1}\right){ }_{P}^{\left(b_{2}\right)} E_{B}\left(b_{3}\right) S_{B}\left(b_{4}\right)
$$

[Q, discharge, in cubic feet per second; A, drainage area in square miles;

$\mathrm{P}$, (mean annual precipitation, in inches -10 inches); $\mathrm{E}_{\mathrm{B}}$, (mean basin elevation, in feet $-5,000$ feet) per 1,000 feet; $S_{B}$, mean basin slope, in feet per feet; $a, b_{1}, b_{2}, b_{3}, b_{4}$, regression coefficients]

\begin{tabular}{|c|c|c|c|c|c|c|c|}
\hline $\begin{array}{c}\text { Discharge } \\
\text { characteristic } \\
Q\end{array}$ & $\begin{array}{c}\text { Regression } \\
\text { constant } \\
\text { a }\end{array}$ & \multicolumn{4}{|c|}{$\begin{array}{l}\text { Regression coefficient } \\
\text { of basin characteristics }\end{array}$} & $\begin{array}{l}\text { Number } \\
\text { of } \\
\text { stations }\end{array}$ & $\begin{array}{l}\text { Mean stan- } \\
\text { dard error } \\
\text { (in percent) }\end{array}$ \\
\hline Annual mean- & $9.70 \times 10^{-2}$ & 0.888 & & 1.74 & & 54 & 55 \\
\hline October mean- & 2.84 & .806 & & & 1.11 & 54 & 100 \\
\hline November mean-... & 1.83 & .815 & & & 1.13 & 54 & 87 \\
\hline December mean--.- & 1.22 & .872 & & & 1.26 & 54 & 77 \\
\hline January mean--..- & $9.33 \times 10^{-1}$ & .916 & & & 1.34 & 54 & 77 \\
\hline February mean--... & $6.47 \times 10^{-1}$ & .913 & & & .906 & 54 & 77 \\
\hline March mean--...- & $1.24 \times 10^{-1}$ & .861 & 0.502 & & & 54 & 53 \\
\hline April mean-- & $4.22 \times 10^{-2}$ & .961 & 1.13 & & & 54 & 62 \\
\hline May mean- & $1.00 \times 10^{-1}$ & .948 & & 2.24 & & 54 & 55 \\
\hline June mean & $3.17 \times 10^{-2}$ & 1.01 & & 2.76 & & 54 & 98 \\
\hline July mean--- & $1.12 \times 10^{1}$ & .850 & & & 1.68 & 54 & 123 \\
\hline August mean- & 5.13 & .790 & & & 1.32 & 54 & 135 \\
\hline September mean-..- & 3.65 & .811 & & & 1.30 & 54 & 142 \\
\hline 90 percent duration & 1.35 & .902 & & & 2.08 & 54 & 179 \\
\hline 70 percent duration & 1.61 & .863 & & & 1.56 & 54 & 108 \\
\hline 50 percent duration & 2.10 & .855 & & & 1.30 & 54 & 106 \\
\hline 25 percent duration & 6.51 & .862 & & & 1.34 & 54 & 88 \\
\hline 10 percent duration & $2.01 \times 10^{1}$ & 0.857 & & & 1.34 & 54 & 65 \\
\hline 2-year-7-day low--- & 1.87 & .830 & & & 2.22 & 54 & 177 \\
\hline 10-year-7-day low & $\ldots . .$. No & Usable 1 & elation & Define & $d----$ & & \\
\hline 50-year-7-day low & $\ldots-\cdots$ No 1 & Usable I & Relation & Define & $d \cdots \cdots$ & & \\
\hline 2-year-7-day high-- & $5.01 \times 10^{-1}$ & .847 & & 1.89 & & 54 & 51 \\
\hline 10-year-7-day high- & 1.54 & .845 & & 1.58 & & 54 & 41 \\
\hline 50-year-7-day high- & 3.24 & .834 & & 1.36 & & 54 & 37 \\
\hline 100-year-7-day high & 4.27 & .829 & & 1.28 & & 54 & 38 \\
\hline 2 -year peak & 7.87 & .732 & & .847 & & 51 & 38 \\
\hline 5-year peak--- & $5.39 \times 10^{1}$ & .686 & & & & 51 & 42 \\
\hline 10 -year peak- & $6.94 \times 10^{1}$ & .685 & & & & 51 & 41 \\
\hline 25-year peak-..... & $9.11 \times 10^{1}$ & .683 & & & & 51 & 41 \\
\hline 50-year peak-..... & $1.09 \times 10^{2}$ & .682 & & & & 51 & 43 \\
\hline 100 -year peak-.... & $1.28 \times 10^{2}$ & .680 & & & & 51 & 45 \\
\hline 200-year peak-....- & $1.49 \times 10^{2}$ & .679 & & & & 51 & 47 \\
\hline 500-year peak-..... & $1.79 \times 10^{2}$ & .677 & & & & 51 & 52 \\
\hline
\end{tabular}

Note: The results for stations used in this study suggest that the peak-flow models for the southwest region tend to overestimate peak flow by about 25 to 100 percent when site elevation is lower than about 5,500 . 
Table 8.--Summary of regression relations for the northwest region Model: $Q=\mathrm{aA}^{\left(\mathrm{b}_{1}\right)} \mathrm{P}^{\left(\mathrm{b}_{2}\right)} \mathrm{E}_{\mathrm{B}}\left(\mathrm{b}_{3}\right) \mathrm{S}_{\mathrm{B}}\left(\mathrm{b}_{4}\right)$

[Q, discharge, in cubic feet per second; $A$, drainage area in square miles; $\mathrm{P}$, (mean annual precipitation, in inches -10 inches); $\mathrm{E}_{\mathrm{B}}$, (mean basin elevation, in feet $-5,000$ feet) per 1,000 feet; $S_{B}$, mean basin slope, in feet per feet; $a, b_{1}, b_{2}, b_{3}, b_{4}$, regression coefficients ]

\begin{tabular}{|c|c|c|c|c|c|c|c|}
\hline \multirow{2}{*}{$\begin{array}{c}\text { Discharge } \\
\text { characteristic } \\
Q\end{array}$} & \multirow{2}{*}{$\begin{array}{c}\text { Regression } \\
\text { constant } \\
\text { a }\end{array}$} & \multicolumn{4}{|c|}{$\begin{array}{l}\text { Regression coefficient } \\
\text { of basin characteristics }\end{array}$} & \multirow{2}{*}{$\begin{array}{l}\text { Number } \\
\text { of } \\
\text { stations }\end{array}$} & \multirow{2}{*}{$\begin{array}{l}\text { Mean stan- } \\
\text { dard error } \\
\text { (in percent) }\end{array}$} \\
\hline & & $\mathrm{b}_{1}$ & $\mathrm{~b}_{2}$ & $\mathrm{~b}_{3}$ & $\mathrm{~b}_{4}$ & & \\
\hline Annual mean- & $2.05 \times 10^{-2}$ & 0.973 & & 2.63 & & 69 & 56 \\
\hline October mean--- & $2.95 \times 10^{-3}$ & 1.01 & & 3.13 & & 69 & 74 \\
\hline November mean--.-- & $2.86 \times 10^{-3}$ & 1.05 & & 2.92 & & 69 & 71 \\
\hline December mean--.- & $2.78 \times 10^{-3}$ & 1.05 & & 2.84 & & 69 & 71 \\
\hline January mean--.-- & $2.79 \times 10^{-3}$ & 1.05 & & 2.77 & & 69 & 71 \\
\hline February mean---- & $4.53 \times 10^{-3}$ & 1.05 & & 2.45 & & 69 & 67 \\
\hline March mean--... & $2.72 \times 10^{-2}$ & 1.04 & & 1.44 & & 69 & 62 \\
\hline April mean- - & $1.08 \times 10^{-1}$ & .916 & 0.804 & & & 69 & 67 \\
\hline May mean--- & $1.44 \times 10^{-1}$ & .878 & 1.17 & & & 69 & 85 \\
\hline June mean- & $9.59 \times 10^{-3}$ & 1.04 & & 3.76 & & 69 & 88 \\
\hline July mean-- & $8.28 \times 10^{-4}$ & 1.07 & & 4.58 & & 69 & 77 \\
\hline August mean- & $8.13 \times 10^{-4}$ & 1.01 & & 4.21 & & 69 & 92 \\
\hline September mean--- & $9.96 \times 10^{-4}$ & 1.00 & & 3.89 & & 69 & 95 \\
\hline 90 percent duration & $1.00 \times 10^{-4}$ & 1.05 & & 4.81 & & 69 & 137 \\
\hline 70 percent duration & $1.37 \times 10^{-3}$ & 1.04 & & 3.34 & & 69 & 78 \\
\hline 50 percent duration & $3.33 \times 10^{-3}$ & 1.02 & & 3.02 & & 69 & 63 \\
\hline 25 percent duration & $1.04 \times 10^{-2}$ & 1.02 & & 2.80 & & 69 & 56 \\
\hline 10 percent duration & $4.30 \times 10^{-2}$ & .989 & & 2.77 & & 69 & 74 \\
\hline 2-year-7-day low--- & $4.89 \times 10^{-4}$ & 1.03 & & 4.34 & .766 & 68 & 149 \\
\hline 10-year-7-day low & $\cdots--N$ & Usable & Relation & Defined & ----- & & \\
\hline 50-year-7-day low & $-\cdots--$ No & Usable & Relation & Defined & $-\cdots-$ & & \\
\hline 2-year-7-day high-- & $1.94 \times 10^{-1}$ & .875 & 1.26 & & & 69 & 90 \\
\hline 10-year-7-day high- & $6.23 \times 10^{-1}$ & .843 & 1.09 & & & 69 & 70 \\
\hline 50-year-7-day high- & 1.15 & .825 & .990 & & & 69 & 65 \\
\hline 100-year-7-day high & 1.42 & .818 & .959 & & & 69 & 66 \\
\hline 2-year peak- & $7.95 \times 10^{-1}$ & .820 & 1.00 & & & 67 & 71 \\
\hline 5-year peak--- & 1.86 & .794 & .871 & & & 67 & 65 \\
\hline 10-year peak---- & 2.86 & .781 & .802 & & & 67 & 63 \\
\hline 25-year peak--.-- & 4.45 & .768 & .732 & & & 67 & 63 \\
\hline 50-year peak--..- & 5.90 & .759 & .686 & & & 67 & 63 \\
\hline 100 -year peak--.-- & 7.54 & .752 & .646 & & & 67 & 63 \\
\hline 200-year peak-.-- & 9.49 & .745 & .609 & & & 67 & 65 \\
\hline 500-year peak-...- & $1.24 \times 10^{1}$ & .737 & .565 & & & 67 & 67 \\
\hline
\end{tabular}




\begin{abstract}
"Weighted estimates are used for unregulated streams to reduce the time-sampling error that may occur in a station flood-frequency estimate. This time-sampling error is associated with the length of record for a station. A station with a short period of record may have a large time-sampling error because its record may not be representative of the actual flood history of the site which would be based on a large number of years. The observed period of record at a station has the possibility of falling within a wet or dry climatic cycle. The weighted estimate of flood frequency should be a better indicator of the true values because the regression estimate is an average of the flood histories of many gaging stations over a long period of time" (Thomas and Lindskov, 1983).
\end{abstract}

The weighting procedure to use for peak discharge in this report is described by Sauer (1974). This procedure weights the station flood frequency and the regression estimate of flood frequency by the years of record at the station and the equivalent years of record of the regression estimate. The following equation should be used:

$$
Q_{T(w)}=\frac{{ }_{T(s)} \times N+Q_{T(r)} \times E}{N+E}
$$

where $\mathrm{Q}_{\mathrm{T}(\mathrm{w})}=$ The weighted discharge, in cubic feet per second, for recurrence interval T-years;

$\mathrm{Q}_{\mathrm{T}(\mathrm{s})}=$ the station value of the flood based on the historical record, in cubic feet per second, for recurrence interval T-years (from Richter and others, 1984);

$\mathrm{N}=$ the number of years of station data used to compute $\mathrm{Q}_{\mathrm{T}(\mathrm{s})}$;

$\mathrm{Q}_{\mathrm{T}(\mathrm{r})}=$ the regression estimate of the flood, in cubic feet per second, for recurrence interval T-years; and

$\mathrm{E}=$ the equivalent years of record for $\mathrm{Q}_{\mathrm{T}(\mathrm{r})}=10$ years (U.S. Water Resources Council, 1981, p. 21).

The Water Resources Council's (U.S. Water Resources Council, 1981, p. 21) recommendation for equivalent years of record only pertains to the 100-year flood; therefore, the assumption for this study is that the equivalent years of record $=10$ years applies to other recurrence intervals.

\title{
Ungaged Sites
}

This method consists of using the regional relations shown in tables $5-8$. Hydrologic characteristics at ungaged sites can be computed by one of the following procedures: Procedure 1 is for sites where the regression relations for one region are used. Procedure 2 is for sites that are near regional boundaries. Procedure 3 is for sites that are near state boundaries. 
Procedure 1 is used when the entire drainage area of a basin falls in a single hydrologic region (see $\mathrm{pl} .1$ ).

Procedure 2 is used when the basin drainage area upstream from the site of interest crosses over a hydrologic region boundary. Where a site is near a regional boundary, the estimates of that particular characteristic can be quite different depending on the regional relation used. Therefore, a weighting procedure is recommended that utilizes the regression relations for both regions whereby each estimate is weighted by the percentage of the drainage area that lies in each region, and then the two are summed. For example, if the 10-year peak discharge is to be determined at a site where 50 percent of the drainage area lies in the mountain region and 50 percent of the drainage area lies in the northwest region, then the 10-year peak discharge is computed using the appropriate relation $\left(\mathrm{P}_{10}\right)$ for the northwest region multiplied by 0.50 and the appropriate relation $\left(\mathrm{P}_{10}\right)$ for the mountain region multiplied by 0.50 . The two results then are added together to get the estimate of the 10-year peak discharge at the ungaged site.

Procedure 3 applies to sites where the upstream drainage area of the site crosses a state line. Studies presenting relations for peak discharge characteristics have been completed in Utah (Thomas and Lindskov, 1983), Wyoming (Lowham, 1976) and New Mexico (Thomas and Gold, 1982), and each of these states is working on updates which will include relations for estimating additional streamflow characteristics. No major differences are apparent between the results of this study and those for Utah, New Mexico, and Wyoming. However, when a station lies near a state line, streamflow characteristics should be determined by averaging estimates from the relations for both states. For example, to determine the 10-year peak discharge at a site near the Colorado-Wyoming State line, the 10-year peak discharge should be calculated using both Colorado's relation (tables 5-8) and Wyoming's relation (Lowham, 1976); then an arithmetic mean of the two results should be considered the best estimate of the 10-year peak discharge.

\section{Limitations and Accuracy}

The regression relations defined in this study provide estimates of flow in streams where the flow is not significantly altered by regulation, diversion, or other man-made influences. The relations cannot be used to estimate present or future flows in streams in urban areas unless the effects of urbanization on streamflow are insignificant. For example, the relations could be applied to a large stream (large rural drainage) flowing in a natural channel through an urban area.

The mean standard error of the regression relations applies only to flow estimates derived for basins in which the values of the independent variables are within the range of the measurements in the gaged basins. The range and distribution of the variables that appear in the regression relations are shown in figure 2. The accuracy of the regression relations for ungaged basins having climatic or physiographic characteristics outside the range of the gaged basins is untested and therefore is unknown. 

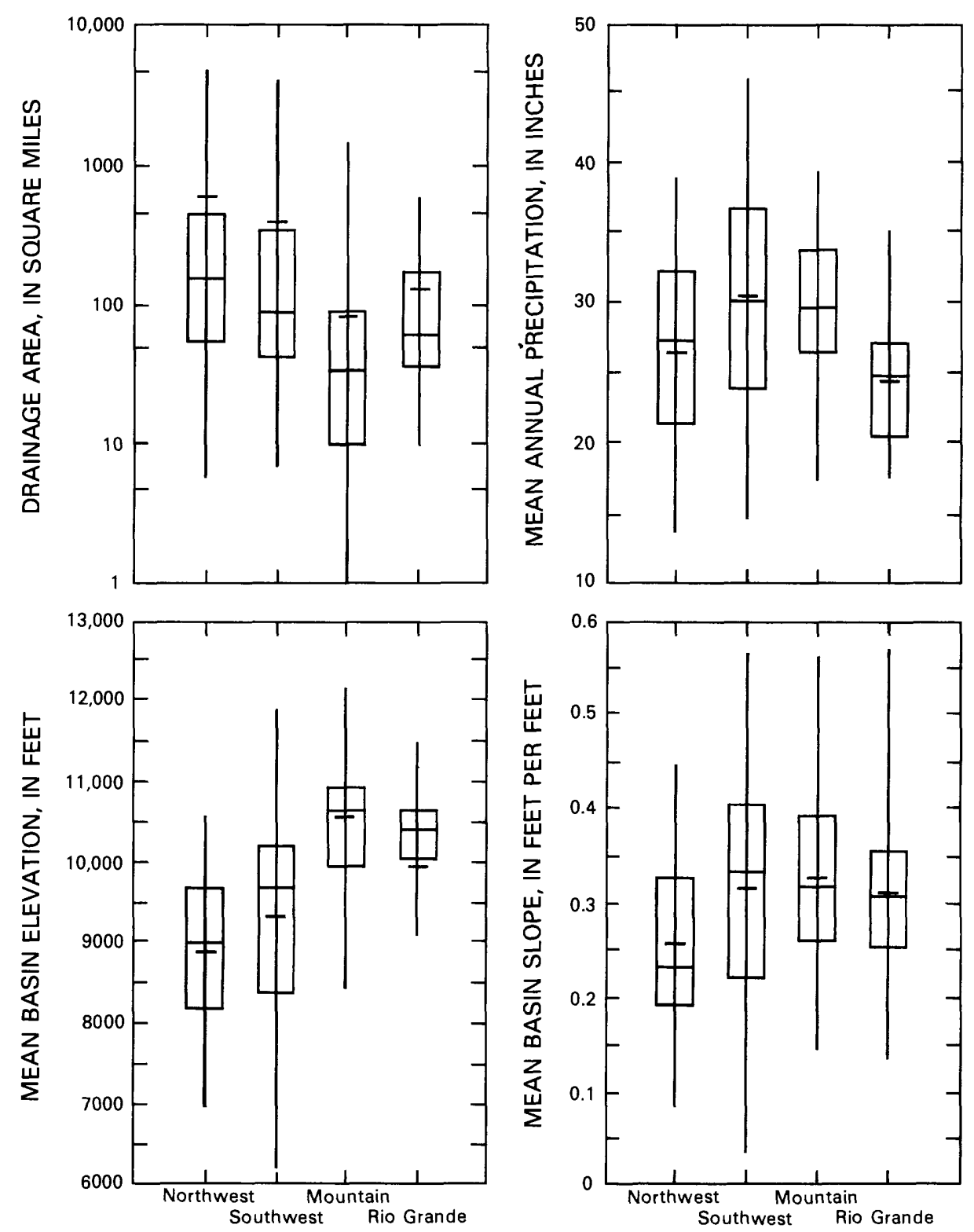

HYDROLOGIC REGIONS

EXPLANATION

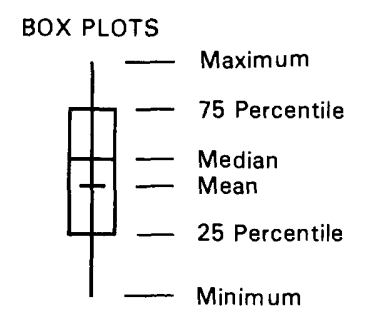

Figure 2.--Range and distribution of basin characteristics used in regression relations for each hydrologic region. 
The accuracy of the regression relations differs between hydrologic regions and specific flow characteristics (fig. 3). Based on averages of the mean standard error for all types of flows excluding minimum 7-day discharges, the accuracy of the predictions decreases in the following order: mountain region, 49 percent; Rio Grande region, 64 percent; northwest region, 73 percent; and southwest region, 73 percent. The regression relations show the smallest mean standard error (about 45 to 70 percent) for estimates of mean annual discharge and flood volumes. The accuracy of the regression relation varies by month for mean monthly discharge but generally is best during November through March, except in the southwest region, where the February through May regression relations show the smallest mean standard error. However, discharges and the range in discharge are generally small during November through March, and comparisons between monthly standard errors (in percent) can be misleading.

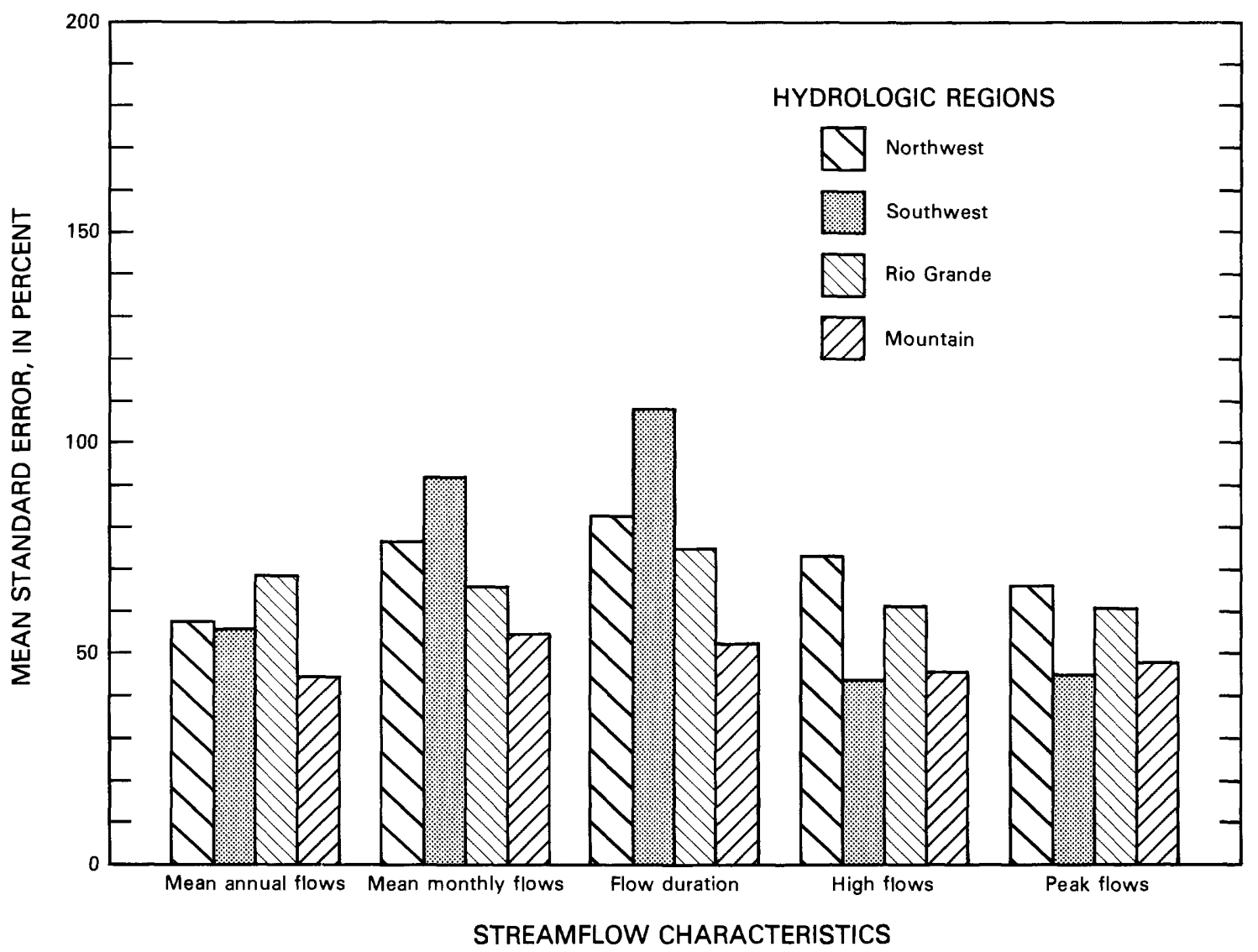

Figure 3.--Mean standard error of regression estimates for selected flow characteristics in each hydrologic region. 
Mean standard errors associated with low-flow relations were commonly in excess of 100 percent, and in five of the nine attempted relations, no usable relation could be defined because of exceedingly high mean standard errors. The difficulty of modeling low-flow volumes reflects large low-flow variability that is not explained by independent variables that can be easily quantified. Low flows can be strongly affected by factors such as structure of the bedrock, depth and permeability of soils, and type and density of vegetation. In addition, even small irrigation diversions can affect low-flow volumes and contribute to the variability of gaged low flows.

For these reasons, most reported attempts at low-flow regionalization encompassing large geographic regions have been unsuccessful (Riggs, 1973). More reliable results generally can be obtained at an ungaged site by relating a series of low flows at an ungaged site to concurrent flows at a nearby gaging station at which the low-flow frequency curve is defined (Riggs, 1965, 1970; Hardison and Moss, 1972). Based on this relation, other low-flow characteristics at the ungaged site can be extrapolated from the frequency curve for the gaged site.

The residuals of the peak-discharge relations in the southwest region indicate that for site elevations lower than about 5,500 ft, the peakdischarge relations tend to overestimate flow by about 50 percent (mean standard error). Because few gaging stations in the southwest region are located below 5,500 ft, the consistency of this bias is uncertain; however, the peak discharge estimates from the regression equations may overestimate flood volumes at other low-elevation sites in this region.

Several guidelines apply to use of the estimates obtained from the regression relations. The mean standard error (tables 5-8) is only an approximate indication of the expected accuracy of the estimate; based on the data from which the relations were developed, the discrepancy between the estimate and actual streamflow will exceed the mean standard error about 30 percent of the time. The large mean standard errors associated with low-flow estimates (tables 5-8) indicate that estimates of low-flow volumes, including minimum 7-day discharge, mean monthly discharge for low-flow months, or flow-durations involving base flow, are only approximate estimates of actual flows. These flow characteristics might be better estimated using other techniques (Riggs, 1970, 1972).

An additional source of error may be associated with estimates of flows having long recurrence intervals. The median length of record in the four hydrologic regions was about 20 years, and the maximum length of record was about 70 years. Length of record has been shown to be the most important single factor affecting the accuracy of streamflow values estimated by the log-Pearson distribution (Benson, 1952; Ott, 1971; Nasseri, 1976).

Because distribution of streamflow-gaging stations on natural-flow channels is not uniform throughout western Colorado (pl. 1), site location is an additional factor to consider in evaluating the accuracy of the regression relations for ungaged basins. Several areas that are physiographically or geologically distinctive and contain few or no gaging stations include the following: 
(1) San Luis Valley (below an elevation of about 8,000 ft);

(2) Uncompahgre Plateau;

(3) Roan Plateau;

(4) North Park and South Park; and

(5) Low-elevation areas (less than about 6,000 ft) in the western part of the northwest hydrologic region.

The lack of streamflow records that met the specifications of this study prevented verification of the hydrologic similarity of these areas to other parts of the hydrologic region in which they occur. When such areas lacking gaging stations were located near hydrologic region boundaries, boundaries necessarily were determined by considering other physical controls on streamflow amounts such as topography, geology, and climatic characteristics.

\section{SUMMARY AND CONCLUSIONS}

Regression relations for estimating streamflow characteristics were developed for western Colorado. Regression relations were determined for 33 flow characteristics, which include mean annual and mean monthly discharges, flow-duration series, peak discharge, and minimum and maximum 7-day discharges of various recurrence intervals. The study area was divided into four hydrologically distinct regions to decrease the variablilty in streamflow caused by differences in basin physiography and climate. Records from 264 stations located in the study area were used to determine relations for the four hydrologic regions.

Drainage area was the most significant variable in all of the streamflow relations. Other significant variables in the regression relations were mean annual precipitation, mean basin elevation, and mean basin slope. The final regression relations include these four basin and climatic characteristics.

It is not recommended that the relations be applied where basin characteristics are outside the range of the data from which the relations were developed or are in local regions of the study area where gaged records of natural flow were not available to develop the relations. The low-flow relations should be used only as an indicator of expected flows because of large mean standard errors associated with the regression estimates of low flows. 
Benson, M.A., 1952, Characteristics of frequency curves based on a theoretical 1000 years record in Dalrymple, Tate, 1960, Flood-frequency analyses: U.S. Geological Survey Water-Supply Paper 1543-A, p. 51-74.

Colorado Climate Center, 1984, Colorado average annual precipitation, 1951-80, 1:500,000 scale map: Colorado Climate Center, Colorado state University, Fort Collins, Colo.

Dalrymple, Tate, 1960, Flood-frequency analyses: U.S. Geological Survey Water-Supply Paper 1543-A, $80 \mathrm{p}$.

Elliott, J.G., Jarrett, R.D., and Ebling, J.L., 1982, Annual snowmelt and rainfall peak-flow data on selected foothills region streams, South Platte River, Arkansas River, and Colorado River basins, Colorado: U.S. Geological Survey Open-File Report 82-426, 86 p.

Fenneman, N.M., 1931, Physiography of the western United states: McGraw-Hill, Inc., New York, $534 \mathrm{p}$.

Hardison, C.H., and Moss, M.E., 1972, Accuracy of low-flow characteristics estimated by correlation of base-flow measurements, in Manual of Hydrology--Part 2, Low-flow Techniques: U.S. Geological Survey WaterSupply Paper 1542-B, p. 35-55.

Hedman, E.R., Moore, D.0., and Livingston, R.K., 1972, Selected streamflow characteristics as related to channel geometry of perennial streams in Colorado: U.S. Geological Survey open-file report, $24 \mathrm{p}$.

Hutchinson, N.E., 1975, WATSTORE--National Water Data Storage and Retrieval System of the U.S. Geological Survey--User's Guide: U.S. Geological Survey Open-File Report 75-426, 791 p.

Jarrett, R.D., and Costa, J.E., 1982, Multidisciplinary approach to the flood hydrology of foothill streams in Colorado, in Johnson, A.I., and Clark, R.A., eds., 1982, International Symposium on Hydrometeorology, Proceedings: American Water Resources Association, p. 565-569.

Livingston, R.K., 1970, Evaluation of the streamflow data program in Colorado: U.S. Geological Survey open-file report, 72 p.

Lowham, H.W., 1976, Techniques for estimating flow characteristics of Wyoming streams: U.S. Geological Survey Water-Resources Investigations 76-112, $83 \mathrm{p}$.

Lystrom, D.J., Rinella, F.A., Rickert, D.A., and Zimmerman, Lisa, 1978, Regional analysis of the effects of landuse on stream-water quality, methodology, and application in the Susquehanna River basin, Pennsylvania and New York: U.S. Geological Survey Water-Resources Investigations $78-12,60 \mathrm{p}$.

Mallows, C.L., 1964, Choosing variables in a linear regression, a graphical aid: Manhattan, Kans., Institute of Mathematical Statistics, May 7-9 Central Regional Meeting. 1973, Some comments on Cp: Technometrics, v. 15, p. 661-675.

Matthai, H.F., 1968, Magnitude and frequency of floods in the United states, Part 6-B, Missouri River basin below Sioux City, Iowa: U.S. Geological Survey Water-Supply Paper $1680,491 \mathrm{p}$.

McCain, J.F., and Jarrett, R.D., 1976, Manual for estimating flood characteristics of natural-flow streams in Colorado: Colorado Water Conservation Board, Technical Manual no. 1, 68 p. 
Nasseri, I., 1976, Regional flow-frequency analysis using multistation stochastic and deterministic models: Palo Alto, Calif., Stanford University, Department of Civil Engineering, Technical Report no. 210, v. 1 .

Ott, R.F., and Linsley, R.K., 1971, Streamflow frequency using stochastically generated hourly rainfall: Palo Alto, Calif., Stanford University, Department of Civil Engineering, Technical Report no. 151, 14 p.

Patterson, J.L., 1964, Magnitude and frequency of floods in the United states, Part 7, Lower Mississippi River basin: U.S. Geological Survey Water-Supply Paper 1681,636 p. 1965, Magnitude and frequency of floods in the United states, Part 8, Western Gulf of Mexico basins: U.S. Geological Survey Water-Supply Paper $1682,506 \mathrm{p}$.

Patterson, J.L., and Somers, W.P., 1966, Magnitude and frequency of floods in the United states, Part 9, Colorado River basin: U.S. Geological Survey Water-Supply Paper 1683, 475 p.

Richter, B.D., Kircher, J.E., Remmers, M.A., and Forst, B.A., 1984, Summary of basin and streamflow characteristics for selected basins in western Colorado and adjacent states: U.S. Geological Survey Open-File Report 84-137, $226 \mathrm{p}$.

Riggs, H.C., 1965, Estimating probability distributions of drought flows: Water and Sewage Works, v. 112, no. 5, p. 153-157. 1970, The transfer value of information collected on representative basins: Wellington, New Zealand, International Association of Scientific Hydrology Publication 96, p. 614-631. 1972, Low-flow investigations: U.S. Geological Survey Techniques of Water-Resources Investigations, bk. 4, chap. B1, 18 p. 1973, Regional analyses of streamflow characteristics: U.S. Geological Survey Techniques of Water-Resources Investigations, bk. 4, chap. B3, $15 \mathrm{p}$.

Sauer, V.B., 1974, Flood characteristics of Oklahoma streams: U.S. Geological Survey Water-Resources Investigations 52-73, $301 \mathrm{p}$.

Statistical Analysis System (SAS) Institute, Inc., 1982, SAS User's Guide: Statistics, 1982 Edition: Cary, N. C., SAS Institute, Inc., 584 p.

Thomas, B.E., and Lindskov, K.L., 1983, Methods for estimating peak discharge and flood boundaries of streams in Utah: U.S. Geological Survey Water-Resources Investigations $83-4129,77 \mathrm{p}$.

Thomas, D.M., and Benson, M.A., 1970, Generalization of streamflow characteristics from drainage basin characteristics: U.S. Geological Survey Water-Supply Paper 1975, 55 p.

Thomas, R.P., and Gold, R.L., 1982, Techniques for estimating flood discharges for unregulated streams in New Mexico: U.S. Geological Survey Water-Resources Investigations Report 82-24, available from National Technical Information Service, Springfield, Va., as PB 82-264 953, 42 p.

U.S. Water Resources Council, 1981, Guidelines for determining flood flow frequency: Hydrology Committee Bulletin 17B, $180 \mathrm{p}$. 